\title{
Diurnal modulation of multivesicular release controls the efficiency of information transmission at a sensory synapse
}

\author{
José Moya-Díaz, Ben James, Federico Esposti, Jamie Johnston and Leon Lagnado* \\ Sussex Neuroscience, School of Life Sciences, University of Sussex, \\ Brighton BN1 9QG, UK \\ *Corresponding author: I.lagnado@sussex.ac.uk
}

\section{Summary}

Neuromodulators adjust sensory circuits to changes in the external world or the animal's internal state and synapses are key control sites for such plasticity. Less clear is how neuromodulation alters the amount of information transmitted through the circuit. We investigated this question in the context of the diurnal regulation of visual processing in zebrafish, focusing on synapses of retinal bipolar cells. We demonstrate that contrast-sensitivity peaks in the afternoon accompanied by an average four-fold increase in the Shannon information transmitted at individual active zones. This increase reflects higher synaptic gain, lower spontaneous "noise" and reduced variability of evoked responses. Simultaneously, an increase in the probability of multivesicular events with larger information content increases the efficiency of transmission (bits per vesicle) by factors of 2-3. This study demonstrates how the potentiation of multivesicular release by neuromodulators can increase the synaptic transfer of information and the efficiency of the vesicle code. 


\section{Introduction}

It has long been understood that the flow of signals through neural circuits is adjusted by neuromodulators ${ }^{1}$. Less clear is how these alter the amount of information that is transmitted through the circuit. Here we investigate this question in the context of visual processing in the retina.

The retina is highly plastic: the input-output relation can adapt within seconds to the recent history of the visual stimulus ${ }^{2,3}$ or, on longer time-scales, to changes in the animal's internal state ${ }^{4,5}$. In diurnal animals, for instance, retinal sensitivity to light is regulated both by the daily light-dark cycle and by intrinsic circadian clocks ${ }^{6-8}$. Key to these adjustments is dopamine, a neuromodulator which is released from amacrine cells in a circadian cycle, varying from a minimum at night, increasing during the day and peaking before dusk ${ }^{6,9}$. But the average luminance of a visual scene is not the variable driving most behaviours related to vision: navigation, finding food and avoiding predators all depend on detection of fast modulations in light intensity. We therefore investigated the diurnal control of temporal contrast processing, focusing on the visual signal transmitted by glutamatergic synapses of bipolar cells.

Bipolar cells are the bridge between the photoreceptors and ganglion cells that deliver the results of retinal processing to downstream circuits. Their synaptic compartments are an important control point for transformations of the visual signal ${ }^{10}$ and contribute to a number of processing tasks, from adaptive gain control to temporal filtering and the coding of motion, colour, orientation and direction ${ }^{3,11-13}$. Bipolar cells are similar to other sensory neurons, such as photoreceptors, sensory hair cells and electroreceptors, in transmitting information through ribbon synapses containing specialized structures that supply vesicles to the active zone ${ }^{14}$. These sensory synapses do not always operate as Poisson machines in which vesicles are released independently but also signal through multivesicular release (MVR), where the fusion of two or more vesicles is co-ordinated as a single synaptic event ${ }^{15-17}$. The importance of MVR at a number of sites in the brain is now recognized and it has been suggested 
that it might contribute to more complex strategies for transmitting information than modulation of a rate $\operatorname{code}^{18-20}$.

It is difficult to use Shannon's information theory to measure the amount of information transmitted at a synapse because the experimenter needs to observe the symbols conveying the message while also observing or controlling the sensory input $^{21,22}$. This has recently been achieved by multiphoton imaging of the glutamate reporter iGluSnFR ${ }^{23}$ in bipolar cells of larval zebrafish, where it is found that the visual message transmitted from an active zone does not use a simple binary code but is instead composed of a number of symbols, composed of one, two, three or more vesicles released as one event ${ }^{17}$. Here we demonstrate that this strategy of coding by amplitude as well as rate is under diurnal control. Synaptic responses to temporal contrast reach a maximum in the afternoon and are accompanied by a four-fold increase in the Shannon information transmitted at each active zone compared to the morning. Dopamine contributes to this increase in information transfer by reducing several aspects of synaptic "noise" and by increasing the probability of multivesicular events with larger information content, which in turn increases the efficiency of transmission quantified as bits per vesicle.

\section{Results}

\section{Differential regulation of luminance-sensitivity and contrast-sensitivity}

To investigate the diurnal modulation of visual processing in larval zebrafish we began by imaging synaptic activity in bipolar cells with SyGCaMP2 ${ }^{24}$ (Fig. 1A). When animals were placed on a cycle of 14 hours light and 10 hours dark, no significant synaptic responses could be detected at Zeitgeber times 18-0 hours, consistent with previous observations that larvae are blind at subjective night ${ }^{25}$. Visual sensitivity began to recover within 20 mins of light onset, after which responses gradually 

functions (Fig. 1C) allowed the luminance sensitivity to be quantified as the inverse of the irradiance generating a half-maximal response $\left(1 / 1_{1 / 2}\right)$. Over the course of the day, luminance-sensitivity increased gradually over a range greater than 200-fold (Fig. 1D). As in other species, this increase could be explained largely by actions of D2 dopamine receptors because injection of the antagonist sulpiride $(\sim 2 \mu \mathrm{M})$ reduced luminance-sensitivity in the afternoon to levels measured in the morning ${ }^{6}$ (Fig. S1).

A
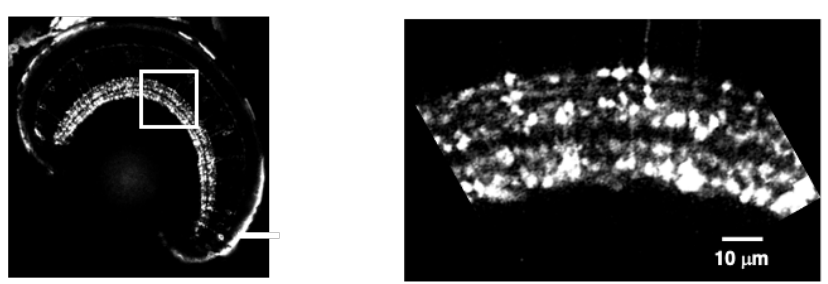

B

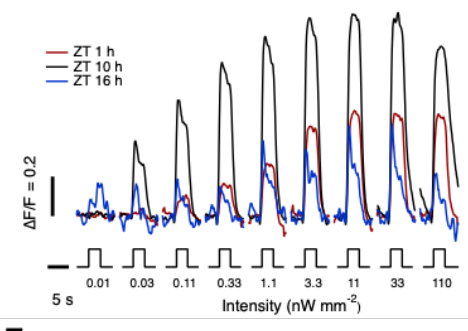

E

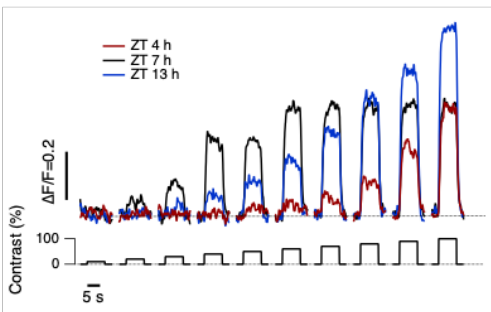

C

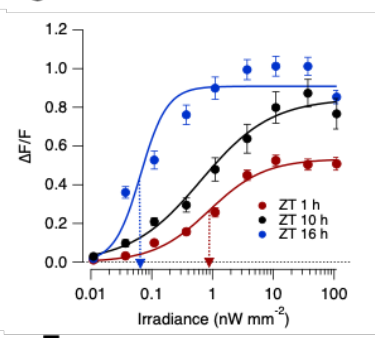

F

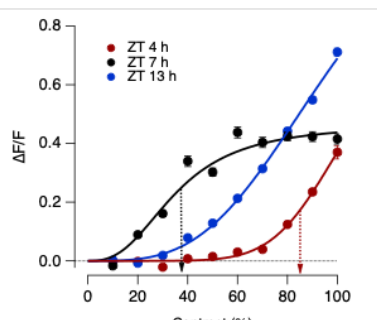

D

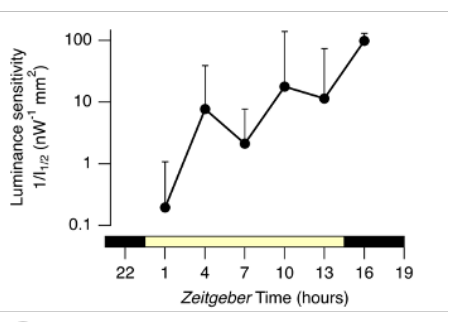

G

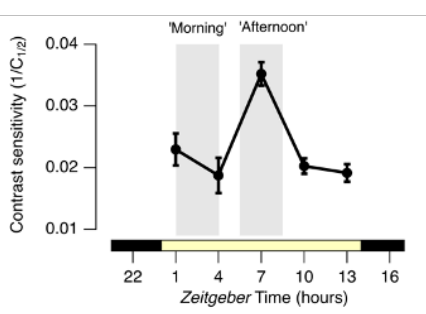

Figure 1: Differential regulation of luminance-sensitivity and contrast-sensitivity

A. Left: Retina of a Ribeye::SyGCaMP2 fish with box over the IPL. Right: expansion of the boxed region of showing terminals of bipolar cells. B. Averaged responses from ON terminals to light steps of different irradiance measured at Zeitgeber time 1, 10 and 16 hours. Note large variations in amplitude and kinetics. Each light step was of $3 \mathrm{~s}(n=535$ terminals from 10 fish). C. Peak response as a function of irradiance for ON terminals in $B$. The smooth lines are Hill functions of the form $R=R_{\max }{ }^{*}\left(I^{h} /\left(l^{h}+I_{1 / 2}{ }^{h}\right)\right)$, where $R$ is the peak response, $I$ is the irradiance, $h$ is the Hill coefficient and $I_{1 / 2}$ is the irradiance generating the half-maximal response. At $Z T=16$ hrs: $R_{\max }=0.91 \pm 0.04 ; h=2.0 \pm 0.2 ; l_{1 / 2}=0.066 \pm$ $0.02 \mathrm{nW} / \mathrm{mm}^{2}$ (dashed blue arrow). At $Z T=10 \mathrm{hrs:} R_{\max }=0.85 \pm 0.06 ; \mathrm{h}=0.8 \pm 0.1 ; \mathrm{l}_{1 / 2}=0.65 \pm 0.18$ $\mathrm{nW} / \mathrm{mm}^{2}$. At $Z T=1$ hrs: $R_{\max }=0.853 \pm 0.02 ; \mathrm{h}=0.9 \pm 0.2 ; \mathrm{l}_{1 / 2}=0.88 \pm 0.18 \mathrm{nW} / \mathrm{mm}^{2}$ (red arrow). D. 
terminals ( $n=535$ and 335 , respectively). The lower bar shows the timing of the light-dark cycle. E. Averaged responses to stimuli of different contrasts measured at Zeitgeber time 4, 7 and $13 \mathrm{hrs}$. F. Peak response amplitude as a function of contrast for terminals shown in $\mathrm{E}$. The smooth lines are Hill functions used to interpolate values of $\mathrm{C}_{1 / 2}$, the contrast generating the half-maximal response. Note the diurnal variations. At $Z T=4$ hrs: $C_{1 / 2}=86 \pm 2 \%$ (dashed red arrow); $h=7.0 \pm 1.2$. At $Z T=7$ hrs: $C_{1 / 2}=35 \pm 2 \%$ (dashed black arrow); $h=2.7 \pm 0.2$. At $Z T=13$ hrs: $C_{1 / 2}=72 \pm 2 \% ; h=3.3 \pm 0.2$. G. Variations in contrast-sensitivity as a function of Zeitgeber time averaged across ON and OFF terminals. Note the peak around $\mathrm{ZT}=7$ hours which is not mirrored in the diurnal variation in luminance sensitivity (D). The grey bars show the periods described as "morning" and "afternoon". All error bars show $\pm 1 \mathrm{SD}$.

The detection of modulations in light intensity (contrast) was also under diurnal control, but with a distinctive time-course (Fig. 1E-G; $5 \mathrm{~Hz}$ full-field stimuli). At ZT $=4$ hours, temporal contrasts below $50 \%$ were barely detected and the half-maximal response $\left(\mathrm{C}_{1 / 2}\right)$ was generated by a contrast of $86 \pm 2 \%$ (Figs. $1 \mathrm{E}$ and $\mathrm{F}$ ). But at $\mathrm{ZT}=$ 7 hours $\mathrm{C}_{1 / 2}$ it fell to $35 \pm 2 \%$ with responses saturated above $50 \%$. When contrast sensitivity $\left(1 / C_{1 / 2}\right)$ was mapped during the course of the day it was relatively constant at ZT 1-5 hours and ZT 9-14 hours but increased to levels $\sim 2.4$-fold higher around ZT $=7$ hours (Fig. 1G). Notably, this peak in the contrast sensitivity of the retinal circuit occurred at a similar Zeitgeber time as the maximum contrast sensitivity measured behaviourally using the optokinetic reflex ${ }^{8,26}$. A qualitatively similar increase in contrast sensitivity was also observed at the retinal output projecting to the optic tectum (Fig.

\section{Diurnal regulation of contrast gain}

To measure transmission of the visual signal in terms of its elementary units synaptic vesicles - we expressed the reporter iGluSnFR ${ }^{23}$ sparsely in bipolar cells (Fig. 2A). Wiener deconvolution of iGluSnFR signals allowed us to count released vesicles

130 (see Methods and Fig. S3; detailed evidence that these methods allow signals to be 131 isolated from individual active zones vesicles has been described ${ }^{17}$ ). Synaptic function was compared over a two-hour period beginning 1 hour after light onset ("morning") with a two-hour period beginning 6 hours later ("afternoon"; Fig. 1G). 
A

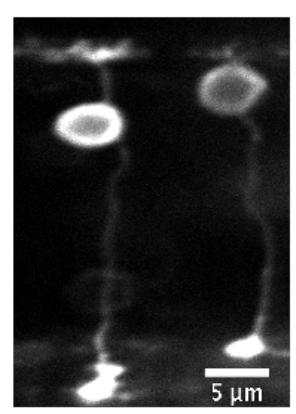

C

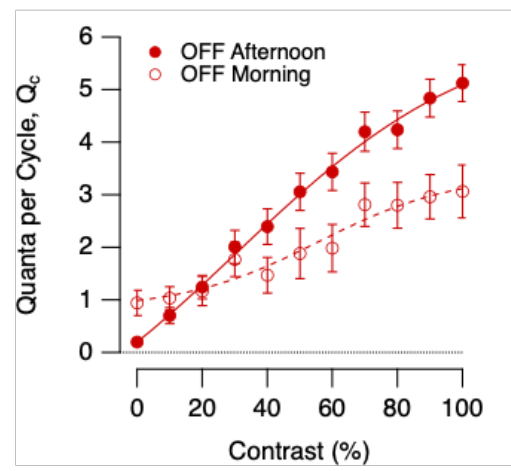

B

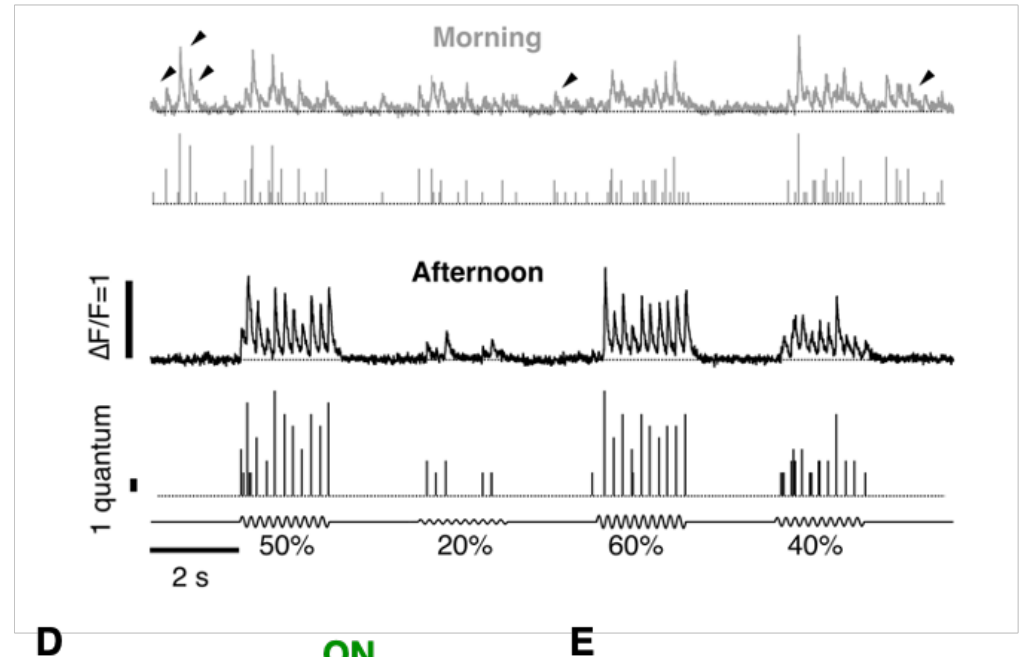

D

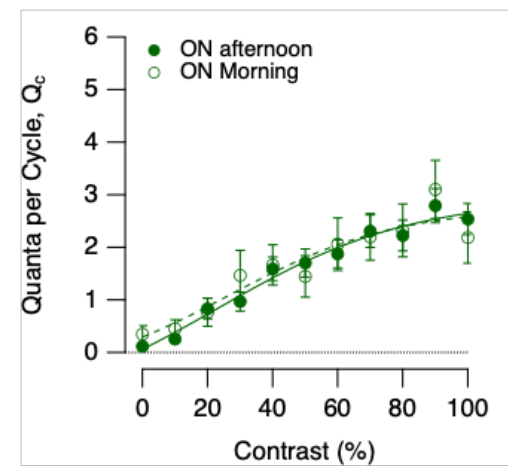

E

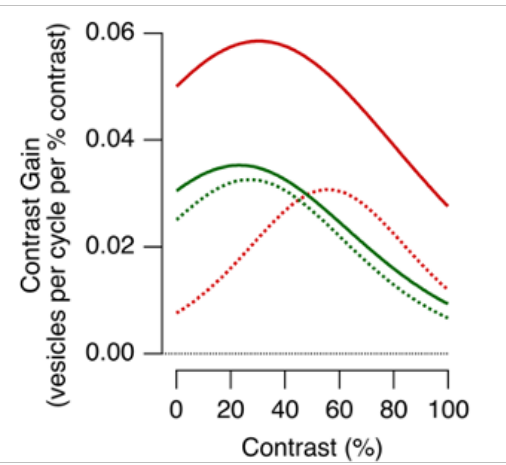

Figure 2. Diurnal modulation of synaptic gain

A. Multiphoton section through the eye of a zebrafish larva (7 dpf) expressing iGluSnFR in a subset of bipolar cells. B. Examples of iGluSnFR signals from an individual OFF synapse elicited using a stimulus of variable contrast modulated at $5 \mathrm{~Hz}(0-100 \%$, full field, sine wave) in the morning (ZT 1-3 hours, grey) and afternoon (ZT 6-9 hours, black). Note the high levels of spontaneous activity in the morning (black arrowheads). In each case the top trace shows the iGluSnFR signal and the lower trace the estimated number of quanta composing each event $\left(Q_{e}\right)$.

C. Average contrast-response functions in OFF bipolar cell synapses in the morning (open circles; $n=20$ synapses) and afternoon (closed; $n=59$ ), where the response $(R)$ was quantified as the average of quanta per cycle $\left(Q_{c}\right)$. The smooth lines are fits of a sigmoid used for smoothing. Note the differences in the shape of the contrast-response functions and in the levels of spontaneous activity (zero contrast). D. Average contrast-response functions in ON bipolar cell synapses in the morning (open circles; $n=12$ synapses) and afternoon (closed; $n=31$ ). There was no significant difference in in the morning relative to afternoon (Chi-square test, $p=0.9999$ ). E. The contrast gain calculated as the derivative of the fits to the contrast-response functions in $C$ and $D$. Note that the maximum contrast discrimination is increased by a factor of $2 x$ in the OFF channel during the 
Examples of glutamate transients at an individual OFF active zone are shown in

156 Fig. 2B. Across a range of contrasts, responses were, on average, larger in the

157 afternoon. We began by measuring the contrast-response function (CRF) simply as

158 the average number of vesicles released per cycle of a $5 \mathrm{~Hz}$ stimulus, choosing this

159 frequency because the integration time of a bipolar cell is $\sim 200 \mathrm{~ms}^{27}$. There was little

160 diurnal modulation of the CRF measured at ON synapses but in the OFF channel the

161 maximum rate of release measured at $100 \%$ contrast increased from $15.25 \pm 2.5$

162 vesicles/s in the morning to $25.5 \pm 1.5$ vesicles/s in the afternoon (Fig. $2 \mathrm{C}$ and D).

163 This increase in synaptic gain was accompanied by an increase in contrast sensitivity,

164 and the combined effects were assessed as the derivative of the CRF ("contrast gain";

165 Fig. 2E). Contrasts in natural visual scenes rarely exceed $40 \%{ }^{12}$ and in the morning 166 this range was signalled best through the ON channel. But in the afternoon the OFF 167 channel became dominant, with contrast gains increasing by factors of 2-6.

169 Dopamine regulates contrast gain

170 To test whether dopamine contributes to diurnal changes in contrast sensitivity we 171 injected agonists or antagonists of dopamine receptors directly into the eye. Fig. 3A 172 shows examples of the output from a synapse imaged in the afternoon, before and 173 after injection of the D1 receptor antagonist SCH 23390 (estimated final concentration 174 of $0.1 \mu \mathrm{M})$. Counteracting the actions of endogenous dopamine reduced the average 175 rate of vesicle release and shifted the CRF such that the maximum contrast gain was 176 achieved at higher contrasts (black points in Fig. 3B and C). Conversely, increasing 177 activation of D1 receptors in the morning by injection of the agonist ADTN $(\sim 0.2 \mu \mathrm{M})$ 178 increased response gain. 
A

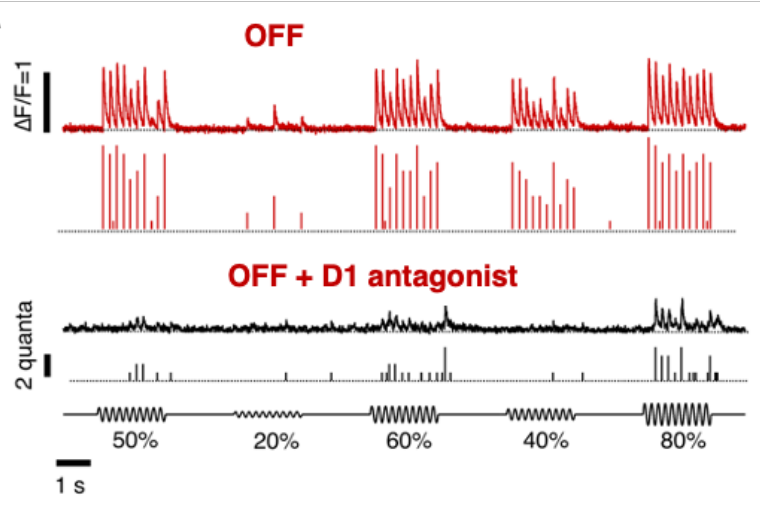

ON

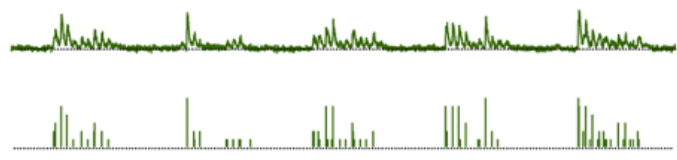

ON + D1 antagonist

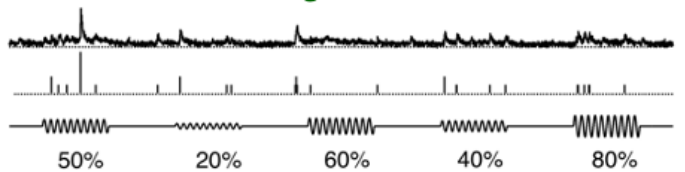

B
C

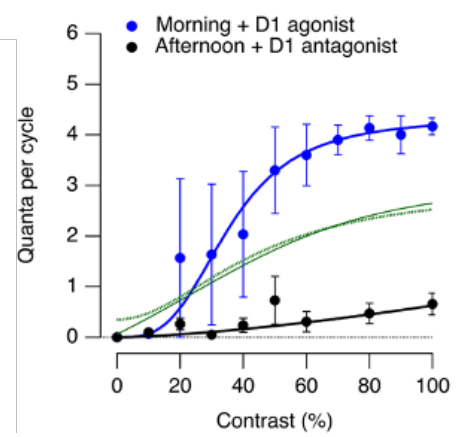

D

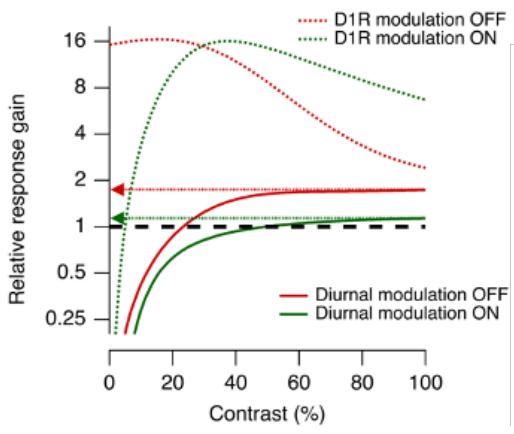

Figure 3. Diurnal changes in dopamine levels modulate synaptic transmission.

A.Examples of iGluSnFR signals recorded in the afternoon from an individual OFF (red trace) and ON (green trace) synapses elicited using a stimulus of variable contrast before and after intravitreal injection of the D1 antagonist, SCH 23390 (black traces; $5 \mathrm{~Hz}$ modulation). Note that SCH 23390 abolished synaptic responses at lower contrasts in ON and OFF synapses. In each case the top trace shows the iGluSnFR signal and the lower trace the estimated $Q_{e}$. B. Average contrast-response functions in OFF bipolar cell synapses after administration of D1 antagonist (black dots) in the afternoon and after administration of the D1 agonist ADTN in the morning (blue dots). Each point shows the mean \pm s.e.m. ( $\mathrm{SCH} 23390, \mathrm{n}=12$ synapses; ADTN, $\mathrm{n}=12$ synapses). Control responses observed in the morning and afternoon are superimposed in the graph (red lines, see Fig. 2C) C. Average contrast-response functions in ON bipolar cell synapses in three conditions: afternoon (green dots), after intravitreal injection of D1 antagonist in the afternoon (black dots) and ADTN in the morning (blue dots). Each point shows the mean \pm s.e.m. (SCH 23390, $n=7$ synapses; ADTN, $n=5$ synapses). Control responses observed in the morning and afternoon are superimposed to the graph (green lines, see Fig. 2D). D. Relative response gain by diurnal modulation and after manipulation of dopaminergic signalling (dashed lines). Note that diurnal modulation of synaptic gain is higher in OFF synapses, whereas dopamine modulates the dynamic range by $\sim 16$ fold-change in ON and OFF synapses.

The dynamic range over which D1 receptors adjusted synaptic gain was calculated as the ratio of the CRFs in the presence of the agonist and antagonist: in both ON and OFF channels the maximum modulation was $\sim 16$-fold, occurring at 
contrasts of $20-40 \%$ (Fig. 3D). But diurnal modulation of gain was narrower than this

202 potential range: 1.7-fold in OFF synapses and 1.1-fold in ON. This difference

203 reflected, at least in part, a gain in the morning that was at least 5-fold higher than that

204 measured with D1R receptors blocked, consistent with dopamine levels that were

205 already high enough to potentiate synaptic transmission (Fig. 3B and C). These

206 manipulations of retinal dopamine receptors caused qualitatively similar changes in the

207 signals that ganglion cells transmit to the optic tectum (Fig. S2).

209 Modulation of synaptic noise and variability

210 How does diurnal modulation of contrast processing affect the information transmitted

211 to ganglion cells? In the framework of information theory ${ }^{21}$, an increase in synaptic

212 gain will tend to reduce uncertainty (and therefore increase information) by causing a

213 larger change in the number of vesicles released when contrast changes. But

214 information is degraded by "noise" that causes responses to vary when the same

215 stimulus is repeated and synapses are a major source of such variability within neural

216 circuits $^{28,29}$. One cause of synaptic noise is the stochasticity of the presynaptic

217 processes that control the fusion of vesicles ${ }^{18}$ and this was a prominent feature of the

218 output from synapses of bipolar cells (Figs. 2B). We distinguished four aspects of

219 synaptic variability and investigated the diurnal modulation of each; i) spontaneous

220 vesicle release (Fig. 4), ii) variability in the number of vesicles released by a stimulus

221 (Fig. 5), iii) variability in the timing of release events i.e how tightly they are time-locked

222 to the stimulus (Fig. 6) and iv) modulation of multivesicular release (Fig. 7). Finally, we

223 calculated how these different aspects of synaptic "noise" combined with changes in

224 contrast gain (Fig. 3) to alter the amount of visual information transferred from

225 individual active zones (Fig. 8). 
A

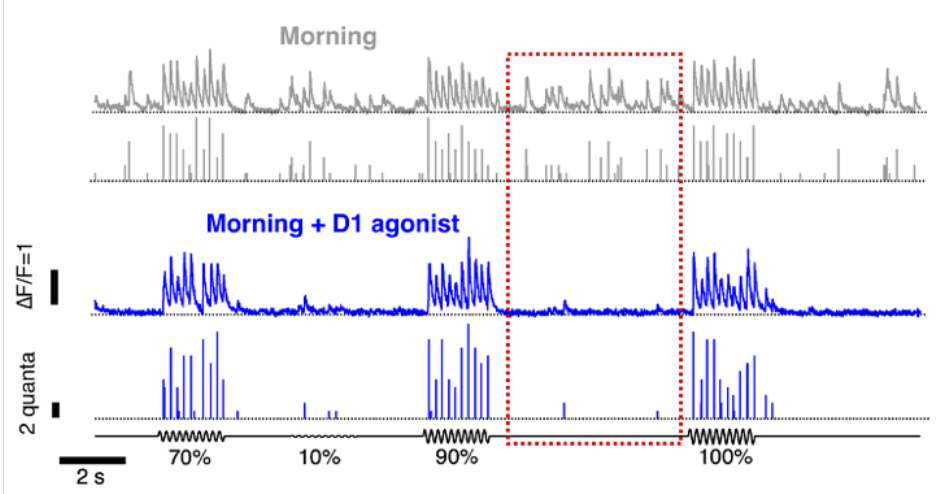

B

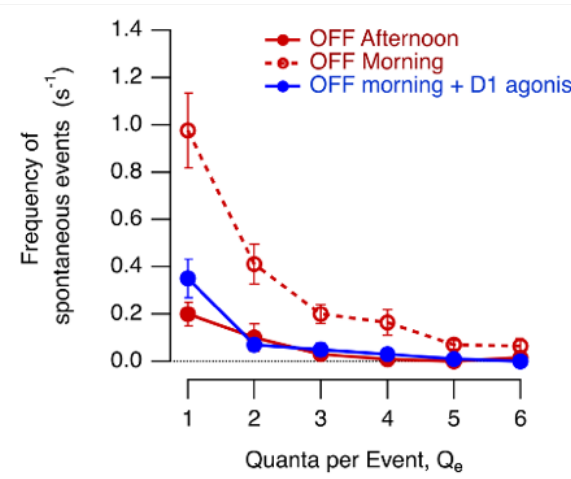

C

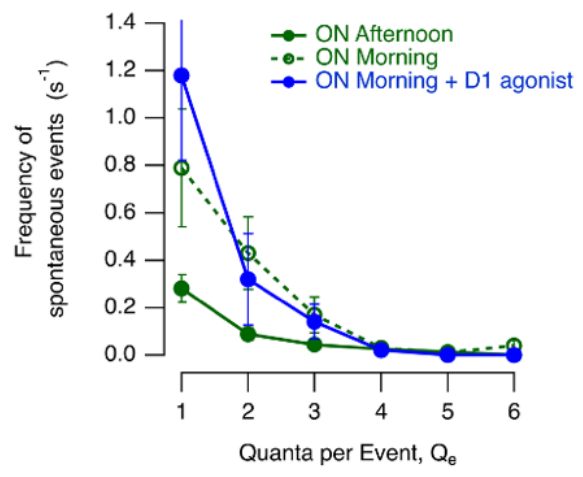

Figure 4. Diurnal modulation of spontaneous synaptic noise

A.Top: Example of iGluSnFR signals from an individual OFF synapse elicited using a stimulus of variable contrast in the morning $(0-100 \%, 5 \mathrm{~Hz}$ modulation). In this example, note the high levels of spontaneous activity that were quantified as the responses elicited at zero contrast (red dashed box). Bottom. Examples of iGluSnFR signals from the same OFF synapse after intravitreal injection of ADTN. Note the increase in amplitude and frequency of events and the reduction of spontaneous activity. In each case the top trace shows the iGluSnFR signal and the lower trace the estimated $Q_{e}$. B. Quantification of spontaneous events composed by different $Q_{e}$ in OFF synapses in the morning, morning + ADTN and afternoon (OFF morning, $n=20$ synapses; OFF morning + ADTN= 12 synapses; OFF afternoon, $n=24$ synapses). Note the suppression of spontaneous events in OFF synapses after intravitreal injection of ADTN in the morning. C. Quantification of spontaneous events composed by different $Q_{e}$ in $O N$ synapses in the Morning, Morning + ADTN and Afternoon (ON Morning = 12 synapses; ON Morning + ADTN = 5 synapses; ON Afternoon, $n=17$ synapses). Note that spontaneous activity levels were not dramatically altered after administration of ADTN.

\section{i) Spontaneous release}

245 Increases in synaptic gain were accompanied by a decrease in the spontaneous 246 release of vesicles in the absence of a visual stimulus. In the morning, spontaneous 
multivesicular events (Fig. 4B and C). Integrating across events of all amplitudes, the average rate of spontaneous release in OFF synapses was $22.5 \pm 12.5$ vesicles s${ }^{-1}$ in the morning, falling to $5 \pm 1$ vesicles $\mathrm{s}^{-1}$ in the afternoon (Fig. 4B). In ON synapses these values were $9 \pm 4$ vesicles $\mathrm{s}^{-1}$ and $2.5 \pm 1$ vesicles $\mathrm{s}^{-1}$ (Fig. $4 \mathrm{C}$ ). In both channels, therefore, spontaneous noise was $\sim 4$ times lower in the afternoon compared to the morning. Increased activation of D1 receptors suppressed spontaneous release in the morning to levels close to those measured in the afternoon, but only in OFF synapses (Fig. 4B and C).

ii) Variability in stimulus-evoked responses.

When recording neural responses as spikes, the Fano factor is measured as the ratio of the variance-to-mean of spikes counted in a fixed time-window after a repeated stimulus $^{30,31}$. We calculated the Fano factor of a synapse by counting the number of vesicles released over each cycle of a sinusoidal stimulus (Fig. 5A). In the morning, $F$ was $\sim 2.6$ in both ON and OFF synapses when averaged over a range of contrasts, falling to $\sim 1.6$ in the afternoon (both significant at $p<0.002$, KS test; Fig. 5B-C). The increase in contrast gain and sensitivity in the afternoon (Fig. 2C-D) was therefore also associated with increased reliability of bipolar cell synapses. Notably, the variability of synaptic output was higher than expected for a Poisson process, for which the Fano factor is one. The spike responses of post-synaptic RGCs are less variable, with a Fano factor as low as 0.3 at higher contrasts ${ }^{30}$, likely reflecting the integration of signals from multiple synaptic inputs. Activation of D1 receptors in the morning improved the reliability of synaptic responses to levels similar to those measured in the afternoon (Figs. 5D and E). 
A

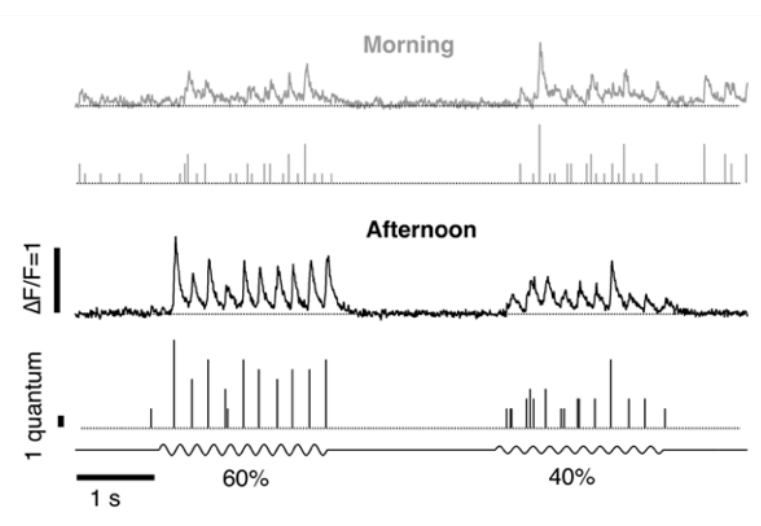

B

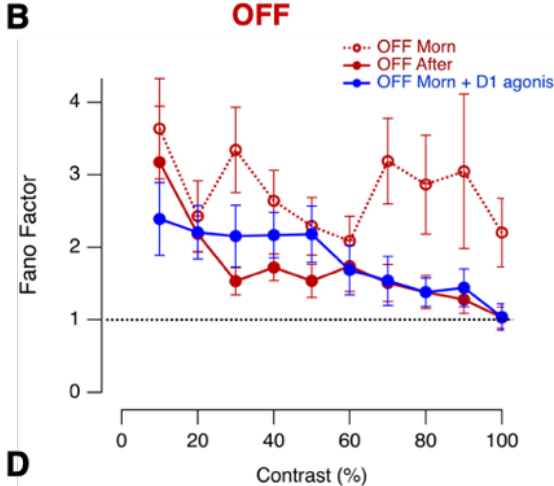

D

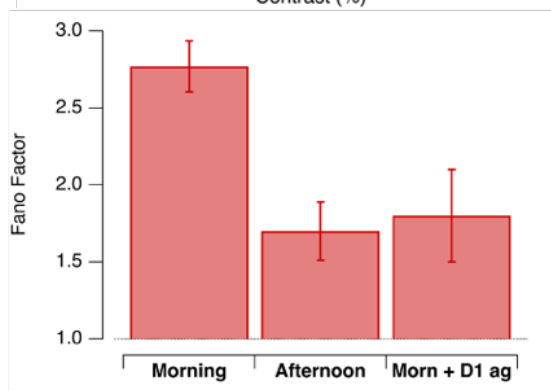

C
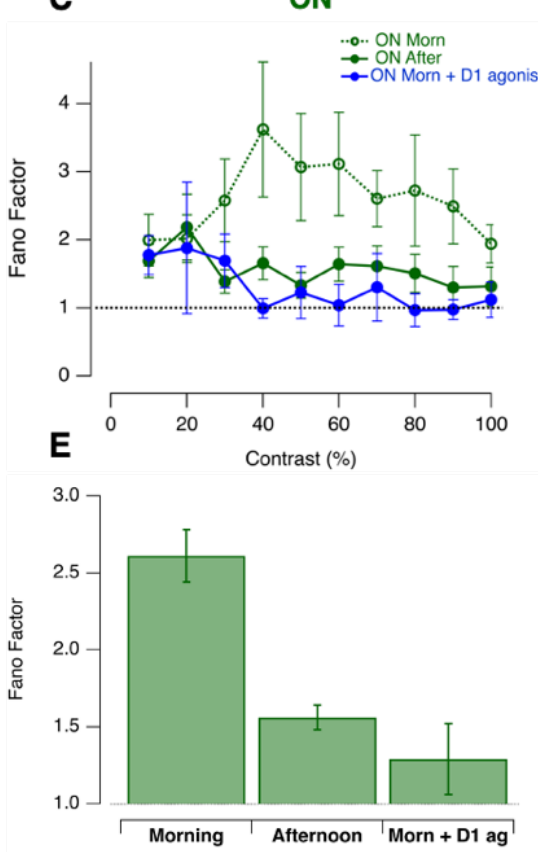

Figure 5. Diurnal changes in the variability of stimulus-evoked responses

A. Examples of iGluSnFR signals from individual OFF synapses in the morning and afternoon. Responses elicited by stimuli of $60 \%$ and $40 \%$ contrast varied from cycle to cycle of the $5 \mathrm{~Hz}$ stimulus. In each case the top trace shows the iGluSnFR signal and the lower trace the estimated $Q_{e}$ B. Variability in the response of OFF synapses calculated as the Fano factor, where each response was quantified as the total number of vesicles released over one cycle at the contrasts shown. Comparison is made between the morning $(n=18)$, afternoon ( $n=27)$ and the morning after injection of ADTN $(n=13)$. C. As in B, but for ON synapses $(n=12,15$ in the three conditions described above. Overall, the average fano factor was significantly higher in the 
A

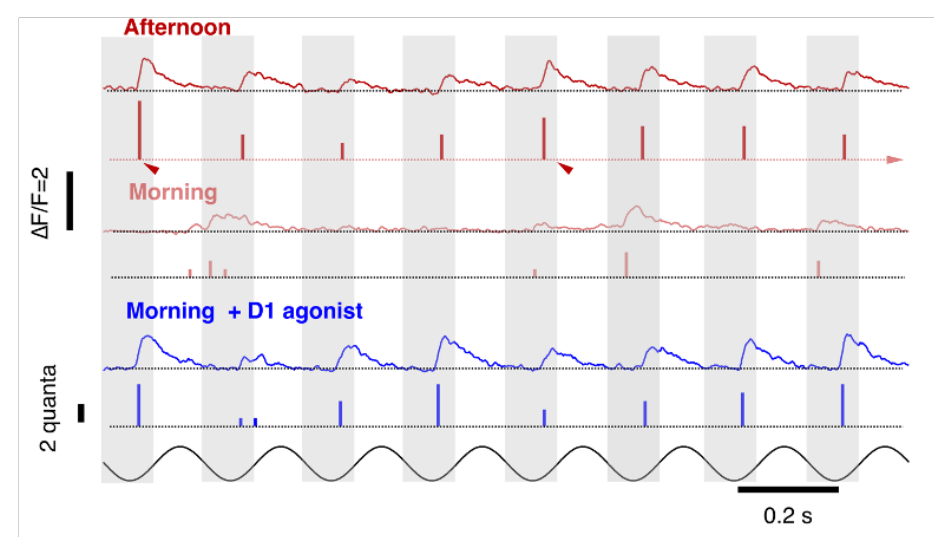

B

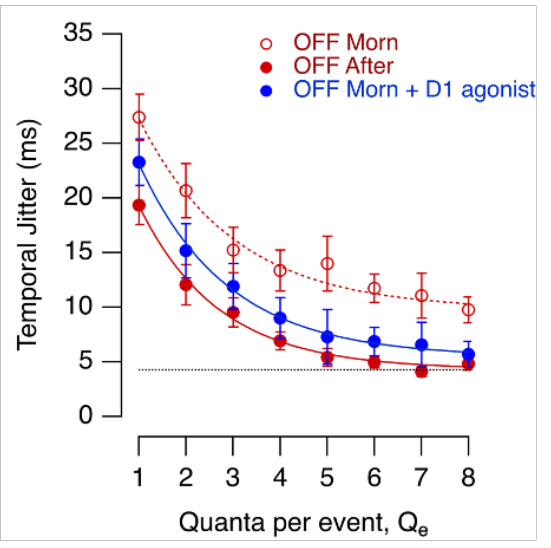

C

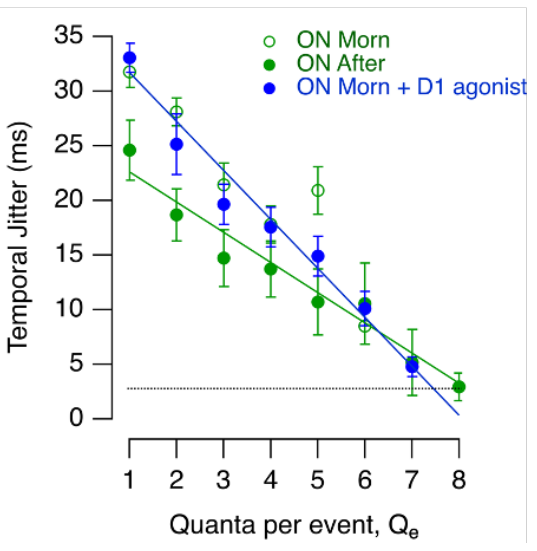

Figure 6. The temporal precision of MVR is under diurnal control in the OFF channel

A. Example recordings from two OFF synapses stimulated at $60 \%$ contrast in three conditions: afternoon (top, black trace), morning (middle, red trace) and after intravitreal injection of ADTN in the morning (bottom, blue trace). Morning and morning + ADTN synaptic responses are from the same synapse. The modulation in intensity ( $5 \mathrm{~Hz}$, sine wave) is shown below. Arrowheads highlight events occurring at different phases of the stimulus, with less variation with events composed for 4 or more quanta in the afternoon and after administration of ADTN in the morning. In each case the top trace shows the

B. Temporal jitter of events composed of different numbers of quanta in OFF synapses in the afternoon (red dots ; $\mathrm{n}=24$ synapses); Morning (open red dots; $n=19$ synapses) and Morning + ADTN (blue dots, $n=16$ ). Note that during the morning events composed by multiple quanta were less phase-locked to the stimuli in comparison to the afternoon. Activation of D1 receptors had a significant effect on release of multiquantal events. Events composed by 5 or more quanta jittered by $\sim 7 \mathrm{~ms}$, similar to values observed in the afternoon. The solid lines describing these relations in the three conditions are better described by a single exponential decay function of the form $\left.y_{0}+A_{\exp }\left(\left(-\left(x-x_{0}\right) / \tau\right)\right)\right)$ with $y 0=4.23 \pm 1.2$ and $A=27 \pm 7$ in the afternoon; $y 0=9.77 \pm 1.4$ and $A=$ $28.64 \pm 5.6$ in the morning and $y 0=5.45 \pm 1.3, A=30 . \pm 6.1$ after activation of $D 1$ receptor in the morning. C. Temporal jitter of events composed by different numbers of quanta measured in ON synapses in the afternoon (green dots; $n=14$ synapses) during the (open green dots; $n=10$ synapses) and during Morning + ADTN, (blue dots; $n=6=$ synapses). Activation of $D 1$ receptor did not have a significant effect in the temporal precision in the $\mathrm{ON}$ channel. The relationship observed in the morning is better described by a straight line with $\mathrm{a}=34.7 \pm 1.5$ and a slope $=-3.6 \pm 0.5$. 
iii) Temporal jitter.

310 Retinal ganglion cells (RGCs) encode information not just in their spike count but also

311 in the timing of spikes ${ }^{30,32}$. Spike times can vary by just a few milliseconds and this

312 accuracy depends on the precision of excitatory inputs received from bipolar cells ${ }^{33}$.

313 The standard deviation in timing of release events ("temporal jitter") was measured 314 relative to the phase of a $5 \mathrm{~Hz}$ stimulus (60\% contrast; Fig. $6 \mathrm{~A})$ and the larger the 315 release events the more precise it was on average (Fig. 6B-C). In OFF synapses the 316 temporal jitter was 5-8 ms higher in the morning compared to the afternoon for events 317 composed of up to 8 vesicles (Fig. 6B; $p<0.008$, Kolomogorov-Smirnov test). Diurnal 318 modulation of temporal precision was weaker in ON synapses and only significant for 319 events composed of 1-3 vesicles (Fig. 3H; t-test at each $\mathrm{Q}_{\mathrm{e}}$ ). Increasing activation of 320 D1 receptors in the morning reduced temporal jitter in events composed for multiple 321 quanta in OFF synapses ( $p<0.05$; KS test) but not ON (Fig. 6B and C; $p>0.5$ ). 322 Diurnal variations in dopamine therefore modulate the temporal accuracy of vesicle 323 release.

\section{iv) Changes in the distribution of multivesicular events}

Previous studies quantifying the synaptic transfer of visual information have been limited by the inability to monitor individual active zones and used the assumption that vesicles are released according to Poisson statistics ${ }^{34,35}$. But we now know that bipolar cells do not employ a simple rate-code and visual information is also contained in the amplitude of multivesicular events ${ }^{17}$. We therefore tested whether modulation of contrast gain was accompanied by changes in $Q_{e}$, the number of quanta in an event.

A comparison of the distribution of $Q e$ in the morning and afternoon is shown in Fig. 7 for responses to a stimulus of $60 \%$ contrast. In ON synapses, $68 \%$ of release events in the morning were univesicular, falling to $40 \%$ in the afternoon and reflecting a shift in the distribution towards larger events (Fig. 7B; $p<0.05, \mathrm{KS}$ test). This shift towards MVR was fully reversed by antagonizing D1 receptors by injection of $\mathrm{SCH}$ 

$38 \%$ of release events being univesicular but again there was a significant shift towards larger events in the afternoon (Fig. 7D; p<0.02). Blocking the D1 actions of endogenous dopamine had a stronger effect in OFF synapses, increasing the proportion of univesicular events to $66 \%$ in the afternoon (Fig. 7E; $p<0.001$ ). Qualitatively similar modulation of MVR was observed over a range of contrasts from $20 \%$ to $80 \%$ and blocking D1 receptors in the afternoon shifted the distribution back to univesicular release in both ON and OFF channels (Fig. 7D and E). Diurnal variations in dopamine therefore modulate MVR.

A
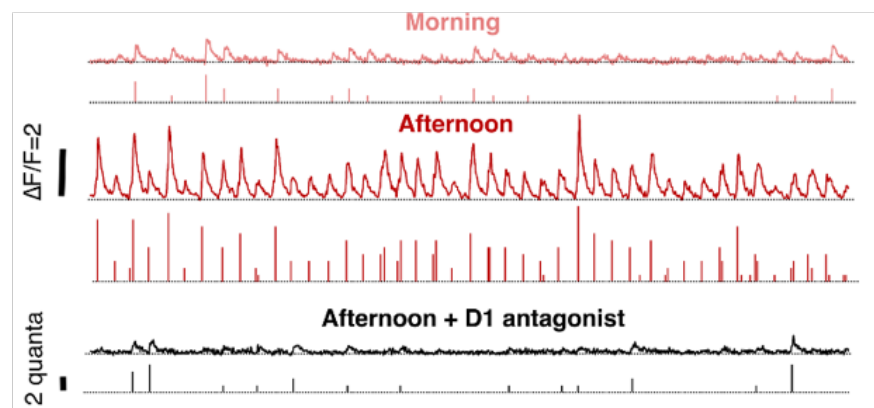

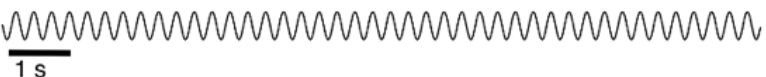

B

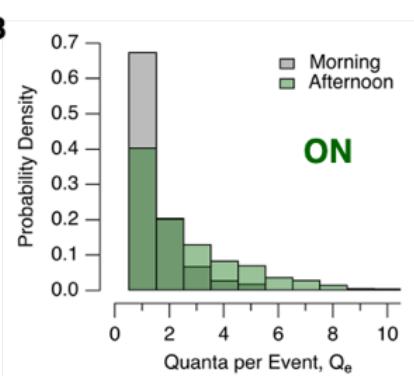

D

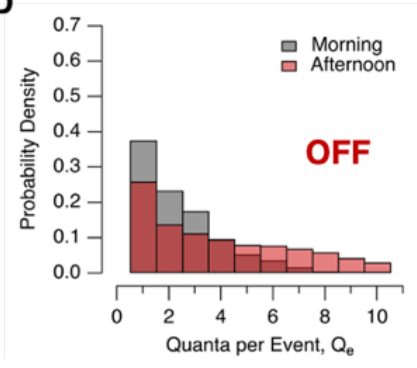

C

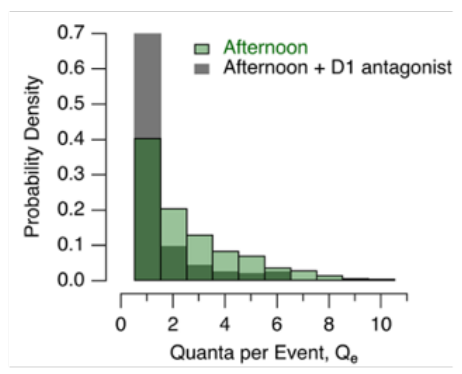

E

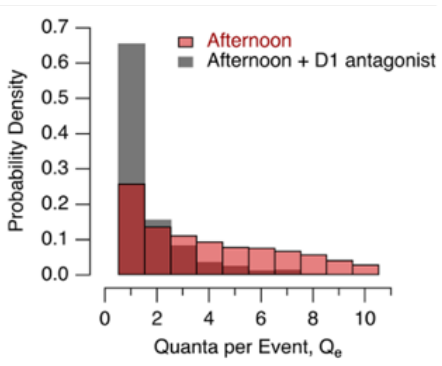

Figure 7. Dopamine contributes to diurnal variations in the distribution of multivesicular events

A. Examples of iGluSnFR signals from individual synapses elicited using $60 \%$ contrast stimulus $(5 \mathrm{~Hz}$, $30 \mathrm{sec}$ ) in the morning (top), afternoon (middle) and afternoon + SCH 23390 (bottom). In each case the top trace shows the iGluSnFR signal and the lower trace the estimated $Q_{e}$. B. Changes in $Q_{e}$ in $O N$ synapses 
( $p<0.059$, Chi-squared test). C. Changes in the distribution of $Q_{e}$ in $O N$ synapses before and after

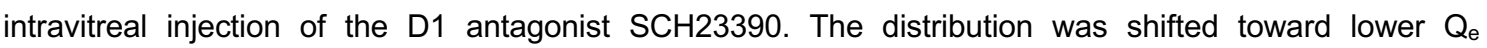
$(p<0.001)$ but was not significantly different to that measured in the morning. $D$. Changes in $Q_{e}$, in OFF synapses in the morning and afternoon. In the afternoon the distribution was shifted toward multiquantal events $(p<0.007)$. E. Changes in the distribution of $Q_{e}$ in OFF synapses before and after intravitreal injection of SCH 23390 in the afternoon. The distribution was shifted toward uniquantal events $(p<0.001)$.

358

\section{Modulation of information encoded at the synapse}

360 How do changes in synaptic gain (Figs. 2-3), noise (Fig. 4-6) and MVR (Fig. 7) combine to alter the amount of visual information transmitted by the synapses of bipolar cells? A larger synaptic signal relative to noise (SNR) will tend to increase the mutual information (I) between the response $(q)$ and the stimulus generating it $(S)$, although the size of the increase will depend on the statistical properties of both signal and noise ${ }^{36}$. In the simple situation where both have a Gaussian distribution I = $0.5 \log _{2}(1+S N R)$. But how should we quantify the synaptic signal? When analyzing the spike code, all events comprise the same symbol and the response can be described as the number of spikes in each of a series of time bins ${ }^{22,36}$. The output from bipolar cells is qualitatively different with a visual stimulus being encoded both by the timing of release events and their amplitudes ${ }^{17}$. We therefore took an approach in which MVR composed of different numbers of vesicles were considered different symbols ${ }^{21,37}$. The mutual information between the response and stimulus was then computed as the average amount of information about the stimulus gained from observing any symbol (see Methods).

The stimulus set $\mathbf{S}$ comprised 12 different contrasts but these were not fixed for each synapse because the contrast sensitivity varied between synapses and between morning and afternoon (Fig. 1E-G). To make allowance for this, we used contrasts

378 spanning $\pm 10 \%$ around $C_{1 / 2}$ measured within the synapse under study immediately before delivering the stimulus set. In the absence of information about the distribution of contrasts normally experienced by a larval zebrafish, a uniform distribution of 
A
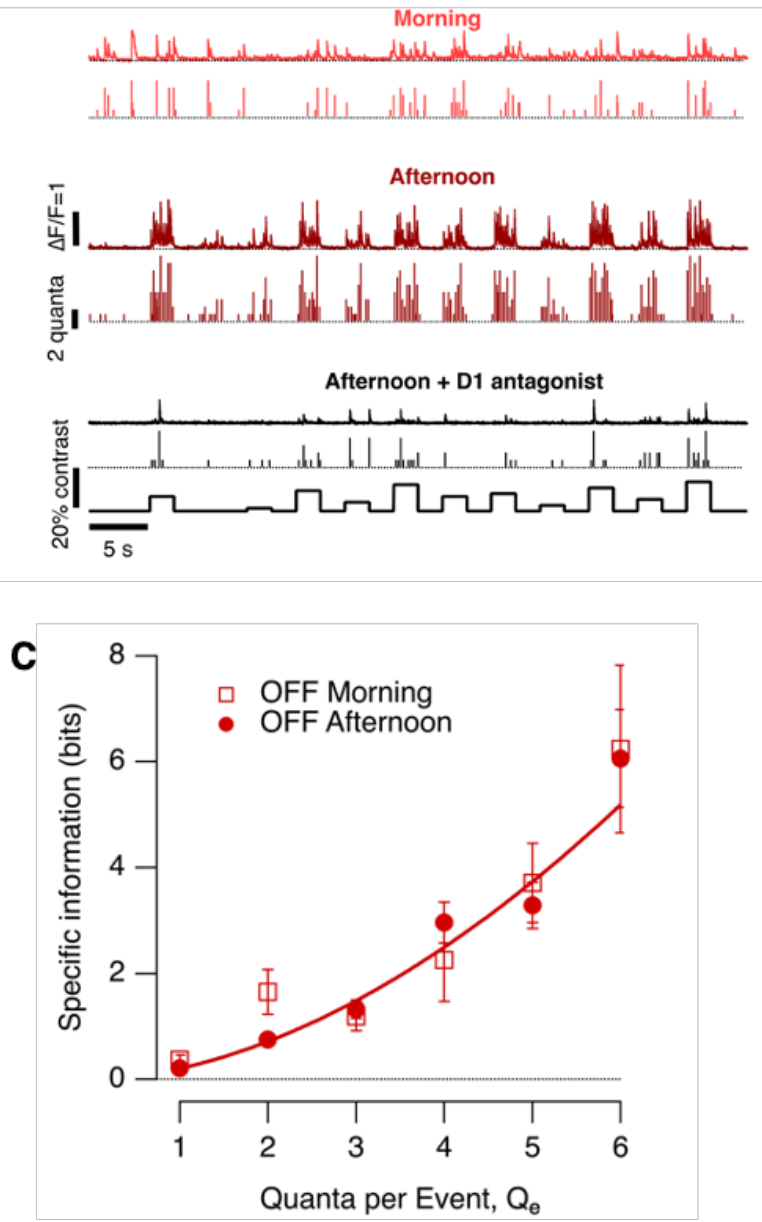

B

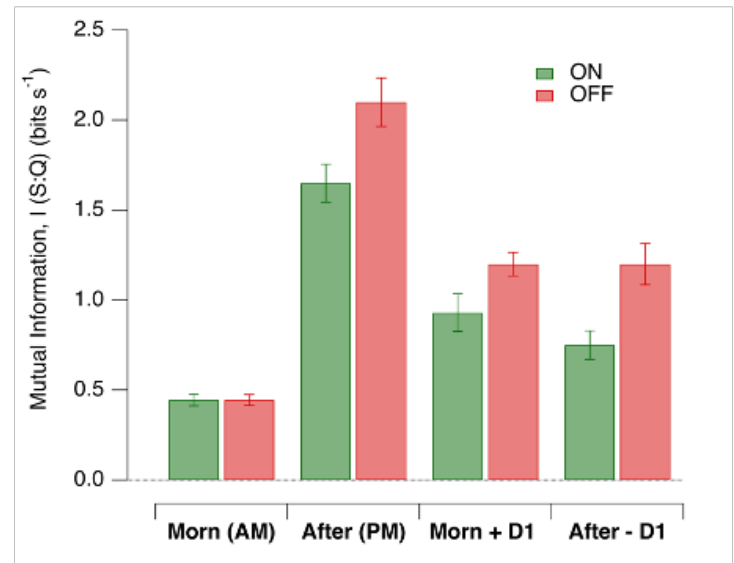

D

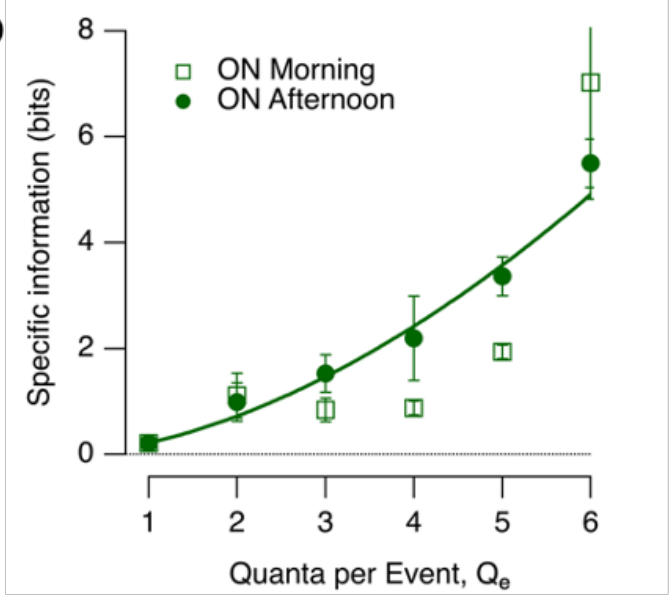

Figure 8. Diurnal changes in the efficiency with which synapses transmit visual information

A. Examples of synaptic responses over 12 different contrasts spanning $\pm 10 \%$ around the contrast eliciting the half-maximal response $\left(C_{1 / 2}\right)$ in the morning (top, light red), afternoon (middle, dark red) and after injection of D1 antagonist SCH 23390 in the afternoon (bottom, black; note the lower frequency and amplitude of release events). In each case the top trace shows the iGluSnFR signal and the lower trace the estimated $Q_{e}$. Each contrast step lasted $2 \mathrm{~s}(5 \mathrm{~Hz})$ and each trace is from a different OFF synapse. B. Mutual information I $(S ; Q)$ in four conditions: (i) morning, (ii) afternoon, iii) morning after injection of ADTN, (iv) afternoon after injection of $\mathrm{SCH} 23390$. C. Specific information $\left(I_{2}\right)$ for events of different quantal content in OFF synapses (33 synapses). The curve describing the relation is a least-squares fit of a power function of the form $i=A Q_{e}{ }^{x}$, with $A=0.20$, and $x=1.81$. D. As $C$, but for ON synapses $(n=13)$. The curve describing the relation is almost identical $(A=0.21$, and $x=1.75)$. 
In the morning, the average mutual information between stimulus and response was almost exactly the same for synapses in the ON and OFF channels $(0.445 \pm$

$398 \quad 0.035$ bits $\mathrm{s}^{-1}$ and $0.455 \pm 0.03$ bits $\mathrm{s}^{-1}$, respectively). In the afternoon mutual 399 information increased through both channels although the increase in OFF synapses $400 \quad(370 \%)$ was significantly larger than in ON $(270 \% ; p<0.001$; Fig. $8 B)$. In OFF synapses, the maximum mutual information of 2.1 bits s$^{-1}$ was associated with average release rate of 2.5 vesicles $\mathrm{s}^{-1}$ around $\mathrm{C}_{1 / 2}$, equivalent to an efficiency around 0.8 bits per vesicle.

Several of the synaptic properties we have analyzed will contribute to the improvement in information transmission in the afternoon, including the increase in synaptic gain (Fig. 2), the decrease in spontaneous noise (Fig. 3) and reduced variability of stimulus-evoked responses (Figs. 5 and 6). These aspects of synaptic transmission were all subject to modulation by dopamine and, consistent with these changes, mutual information in the morning was increased by activation of D1 receptors while in the afternoon it was decreased by antagonizing the effects of endogenous dopamine (Fig. 8B).

These results demonstrate that information transmission through the retina is

414 changes. Antagonizing D1 receptors did not, however, reduce mutual information to 415 levels measured in the morning, leaving open the possibility that other signaling 416 pathways also contribute.

\section{Changes in the efficiency of the vesicle code}

419 The transmission of information using spikes and vesicles is the major consumer of 420 energy in the brain with one estimate being of the order of $\sim 24,000$ ATP molecules per 421 bit $^{38,39}$. The largest part of this energy consumption is taken up by synaptic 422 transmission so a key question becomes the effect of neuromodulation on the 423 efficiency with which vesicles are used to transmit information. Strikingly, the 2.7-fold 
increase in information transmitted through ON synapses in the afternoon (Fig. 8B) was not associated with any change in the average rate of vesicle release (Fig. 2D and E), while the 3.7-fold increase in OFF synapses was associated with only a 2-fold increase in the rate around $\mathrm{C}_{1 / 2}$ (Fig. $2 \mathrm{C}$ and $\mathrm{E}$ ). The diurnal increase in synaptic gain was therefore associated with a 1.4- to 2.7 -fold increase in the average efficiency with which vesicles were used to encode changes in contrast. A comparison can be made with the information transmitted by spikes in RGCs, where the most sluggish cells transmit $\sim 3.5$ bits/spike, while those that fire most briskly encode $\sim 2$ bits/spike ${ }^{40}$. An increase in spike rate is therefore associated with a decrease in the visual information per spike while an increase in vesicle release rate is associated with an increase in information per vesicle.

How is this increase in the efficiency of the vesicle code achieved? The comparison of information transmission with average rates of vesicle release obscures a key aspect of the vesicle code operating in bipolar cells: information about contrast is represented as changes in both the rate and amplitude of release events ${ }^{23}$. This is significant because the distribution of MVR events was also a function of Zeitgeber time and larger events are rarer and carry more specific information ${ }^{17}$, as shown by the supralinear relation between the specific information carried by each synaptic symbol and the number of vesicles it contains (Fig. 8C-D). The diurnal modulation of the efficiency of the vesicle code therefore depends on the shift between univesicular and multivesicular release (Fig. 7B and D).

We also considered the possibility that modulation of retinal processing might be accompanied by changes in the amount of information carried by a given synaptic symbol. The relation between information carried and event amplitude did not, however, change in the afternoon compared to the morning, at least for events composed of 1-6 vesicles (Fig. 8C-D; OFF synapses $p>0.99$; ON synapses $p>0.98$; KS test). 


\section{Discussion}

The plasticity of synapses allows the flow of information through circuits to be modulated $^{1}$ and this study provides a quantitative understanding of this idea in the context of the diurnal control of visual processing in the retina. We find that the daily light-dark cycle alters the transmission of visual information through bipolar cells by factors of $\sim 4$ during daylight hours by adjusting four synaptic properties; the number of vesicles released by a stimulus (Fig. 3), spontaneous synaptic noise (Fig. 4), the variability of stimulus-driven responses (Figs. 5-6) and the balance between univesicular and multivesicular release (Fig. 7). Crucially, the switch in emphasis from univesicular to multivesicular release also increases the amount of information transmitted per vesicle (Fig. 8). Dopamine plays a major role in regulating all these aspects of retinal function although the relative contributions of these mechanisms differed between ON and OFF pathways.

\section{Diurnal modulation of gain}

Dopamine-dependent changes in the synaptic gain of bipolar cells might be caused either by direct modulation of processes within the terminal compartment or by actions on the circuitry in which they are embedded. A direct action is strongly supported by the presence of dopamine receptors (especially D1) on the terminal compartment of bipolar cells ${ }^{41,42}$ and electrophysiological experiments demonstrating that their activation potentiates L-type calcium channels that control vesicle fusion ${ }^{4}$. Dopamine also acts on D2 receptors on cone synapses to potentiate the visual drive to bipolar cells but this mechanism alone does not easily explain the transient increase in contrast-sensitivity in the afternoon given that luminance sensitivity, a much more direct reflection of the strength of cone input, gradually increases throughout the day (cf. Fig. 1B and Fig. 1E). The gain of bipolar cell synapses is also strongly dependent on the inhibitory inputs that the synaptic compartments receives from amacrine cells and the possibility of diurnal modulation of inhibition remains open. 

in luminance ${ }^{7}$ or the appearance of food-related odours ${ }^{4}$. But other neuromodulators, are also released from amacrine cells, including melatonin ${ }^{43}$, Substance $\mathrm{P}^{44}$ and somatostatin $^{45}$ and some of these can antagonize the actions of others ${ }^{44}$. A large number of different proteins control the activity of the retinal circuit and $17 \%$ of genes in zebrafish are under circadian regulation ${ }^{7}$. There is therefore a good possibility that neuromodulators other than dopamine will also act on the synaptic output of bipolar cells, either directly or indirectly, to regulate the visual signal transmitted to ganglion cells.

\section{Diurnal modulation of noise}

491 It has long been appreciated that synaptic noise can reduce the amount of information 492 transmitted through a circuit of neurons ${ }^{28}$. When the retina operates under photopic 493 conditions, for instance, the release of vesicles from bipolar cells adds noise to the 494 signal arriving from cones and therefore causes a loss of information in RGCs ${ }^{46}$. It has 495 been suggested, however, that under other circumstances the noise in synaptic 496 transmission might improve information transmission, such as when stochastic 497 resonance increases the probability of post-synaptic depolarization crossing threshold 498 for spike generation ${ }^{29,47}$. But it seems unlikely that the retina operates under such a 499 regime, given that diurnal increases in synaptic gain went hand-in-hand with a 500 reduction in several sources of noise, including spontaneous release unrelated to a $501 \quad$ stimulus.

502 All the changes in synaptic function that we observed comparing periods in the 503 morning and afternoon were mimicked by manipulating dopamine signalling, indicating 504 that this neuromodulator adjusts information transmission by orchestrating changes in 505 both the signal and the various noise sources that cause it to vary. The balance between modulation of signal and noise was however, strikingly different in the ON channel, where synaptic gain was not under diurnal modulation, compared to the OFF 
508 channel, where both signal and noise were regulated. The processes by which

509 dopamine and other neuromodulators adjust synaptic noise are also likely to involve

510 both direct actions on the synaptic compartment and indirect actions on other

511 components of the retinal circuit.

$513 \quad$ Modulation of multivesicular release

$514 \quad$ MVR is not just a property of ribbon synapses but is also a feature of synaptic 515 transmission in the hippocampus ${ }^{48}$, cerebellum ${ }^{49}$ and somatosensory cortex ${ }^{19}$, where 516 arrival of a spike can often trigger release of two or more vesicles at an active zone. A 517 recent combination of electrophysiology with correlative light-and electron-microscopy 518 has even led to the suggestion that MVR may be a fundamental mode of synaptic 519 transmission throughout the nervous system ${ }^{20}$. It is also recognized that MVR can be 520 adjusted by neuromodulation, for instance through muscarinic acetylcholine receptors 521 in the striatum\{Higley, $2009 \# 221\}$ or GABA $_{B}$ receptors in the cortex\{Chalifoux, 2010 $522 \quad \# 222\}$, athough the implications for information transmission in these contexts is not 523 known. Our study has demonstrated that potentiation of MVR in the retina not only 524 increases the amount of information that a synapse can transmit using vesicles but 525 also the efficiency of coding. It will be interesting to establish how far neuromodulators 526 acting in other parts of the brain alter the efficiency of the information transmission and 527 how far this involves modulation of MVR as compared to the variability and noise that 528 is a feature of central synapses ${ }^{28,29}$. A crucial aspect of these questions will be to 529 understand how switching from univesicular release to multivesicular release alters the $530 \quad$ spike code generated post-synaptically. 


\section{$534 \quad$ Acknowledgements}

535 The authors express many thanks to all the members of Lagnado laboratory for

536 discussion. We also thank Tom Baden for his many insightful criticisms and suggestions.

537 This work was supported by grants to L.L. from the Wellcome Trust (102905/Z/13/Z).

538

$539 \quad$ Author contributions

$540 \quad$ J. M-D. conceived, designed and executed experiments, analyzed results and prepared

541 the manuscript. B. J. carried out analysis and wrote code. F. E. conceived and executed

542 experiments and carried out analysis. J. J. conceived and executed experiments and

543 carried out analysis. L. L. conceived the project, designed experiments, analyzed data,

544 repaired equipment, wrote code and prepared the manuscript.

545

546 


\section{Supplementary Figure 1}

A
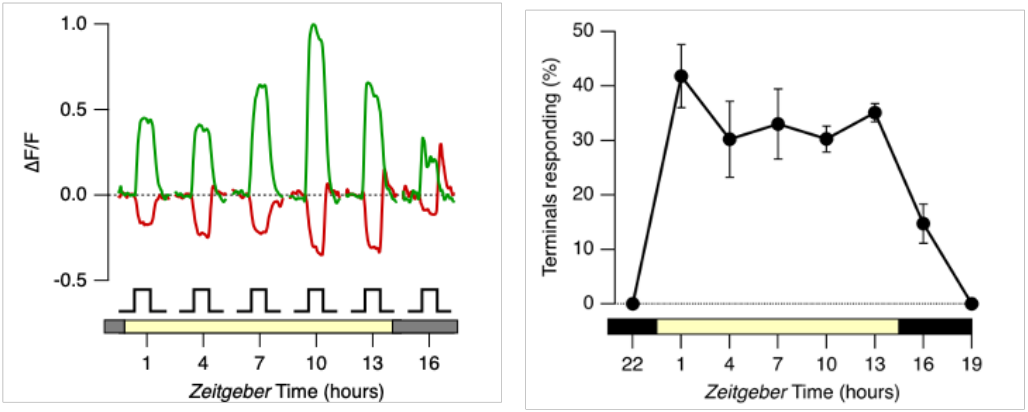

B

ON

c

OFF
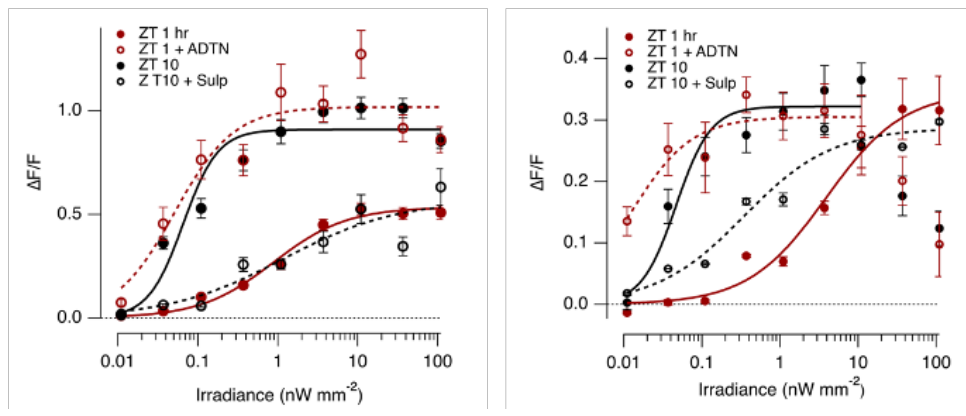

Figure S1. Diurnal changes in luminance sensitivity are co-ordinated by dopamine

A. Left: Averaged SyGaMP2 signals at different Zeitgeber time. ON terminals green and OFF terminals red. Each step of light $\left(10 \mathrm{nW} \mathrm{mm}^{-2}\right)$ lasted $3 \mathrm{~s}$. These averages are only from responsive terminals. Right: the percentage of terminals generating a significant response to the same light step (averaged across both ON and OFF). Bars show SD. B. Effects of manipulating dopamine signalling on luminance sensitivity of the ON channel. Luminance vs. response plots for ON terminals. Red circles compare this function at ZT $1 \mathrm{hr}$ under control conditions (solid circle) and after injection of the non-selective dopamine receptor agonist ADTN ( $\sim 0.2 \mu \mathrm{M}$; open circles). ADTN caused a prompt change in the luminanceresponse function to forms measured at $Z T 10 \mathrm{hrs}$ (solid black circles), increasing $R_{\max }$ from $0.53 \pm 0.02$ to $1.02 \pm 0.07$, and reducing $I_{1 / 2}$ from $0.88 \pm 0.18 \mathrm{nW} \mathrm{mm}^{-2}$ to $0.05 \pm 0.02 \mathrm{nW} \mathrm{mm}^{-2}$ ( $\pm \mathrm{sd}$, as estimated from the fitted Hill function shown). The higher gain and luminance sensitivity at ZT $10 \mathrm{hrs}$ could be explained as an effect of dopamine at D2 receptors, because it was completely reversed by injection of the selective $\mathrm{D} 2$ receptor antagonist sulpiride $\left(\sim 2 \mu \mathrm{M}\right.$; open black circles; $R_{\max }=0.57 \pm 0.13, \mathrm{I}_{1 / 2}=1.16 \pm 1.34 \mathrm{nW} \mathrm{mm}^{-}$ ${ }^{2}$ ). Results collected from $n=535$ terminals from 38 fish. C. Effects of manipulating dopamine signalling on luminance sensitivity of the OFF channel. Comparing control responses at ZT $1 \mathrm{hr}$ and $10 \mathrm{hrs}$ showed a significant reduction in $I_{1 / 2}$ from $3.9 \pm 1.3$ to $0.0128 \pm 0.005$, but without a significant change in $R_{\max }(0.35$ \pm 0.03 vs $0.30 \pm 0.02$ ). ADTN injected at $Z T 1$ caused a prompt increase in luminance sensitivity, reducing $\mathrm{I}_{1 / 2}$ to $0.013 \pm 0.005 \mathrm{nW} \mathrm{mm}^{-2}$. The higher luminance sensitivity at $\mathrm{ZT} 10 \mathrm{hrs}$ could be partly explained as an effect of dopamine at D2 receptors, because injection of sulpiride ( $2 \mu \mathrm{M}$; open black circles) increased $\mathrm{l}_{1 / 2}$ from $0.05 \pm 0.01 \mathrm{nW} \mathrm{mm}^{-2}$ to $0.35 \pm 0.14 \mathrm{nW} \mathrm{mm}^{-2}$. Results collected from $n=355$ terminals from 38 fish. 
A
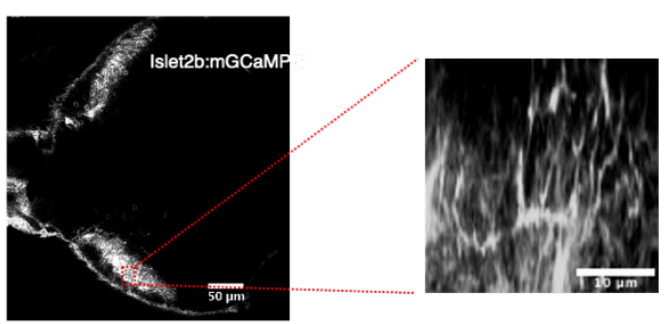

B

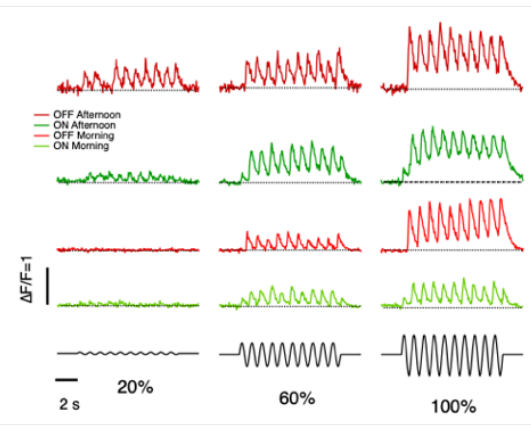

D

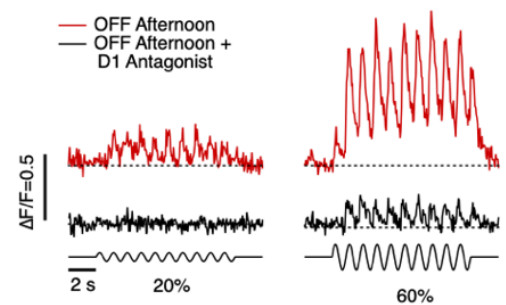
response in both ON and OFF channels.

\section{C}

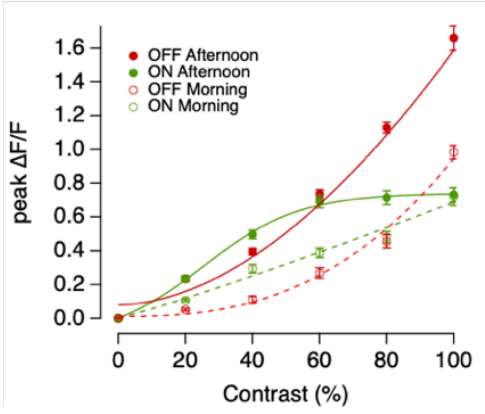

E

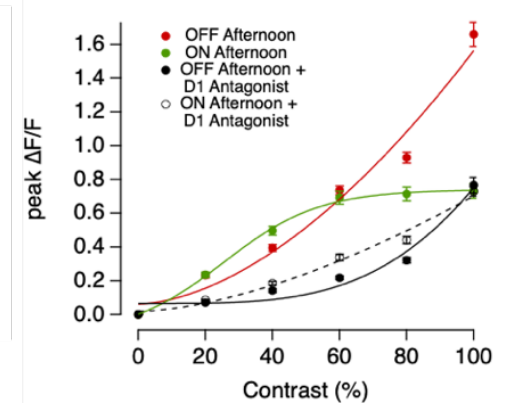

Figure S2: Diurnal changes in the visual signal delivered to the optic tectum

A.Left panel. Multiphoton section through the tectum of a zebrafish larva islet2b::mGCaMP6f (7 dpf) expressing the calcium reporter mGCaMP6f, which labels axons and synaptic terminals of retinal ganglion cells (RGCs). Right panel. Blow-up of the square red shown in the left panel. The image shows the $Z$ plane reconstruction from where the synaptic responses were recorded. B. mGCaMP6f signals from individual ON and OFF RGCs synapses elicited using a stimulus contrasts of $20 \%, 60 \%$ and $100 \%$ modulated at $1 \mathrm{~Hz}$ (full field, sine wave) C. Average contrast-response functions displayed by $\mathrm{ON}$ and OFF RGCs, where the response $(R)$ was quantified as the average of the fluorescence peak amplitudes measured at each cycle of stimulation. Each point shows the mean \pm s.e.m. Note the differences in the magnitude of the responses between OFF RGCs in the morning relative to afternoon. D. mGCaMP6f signals from an individual OFF RGCs before and after intravitreal injection of the D1 antagonist in the afternoon. E. Average contrast-response function displayed by ON and OFF RGCs before and after administration of the D1 antagonist in the afternoon. Note the dramatic decrease in the magnitude of the 


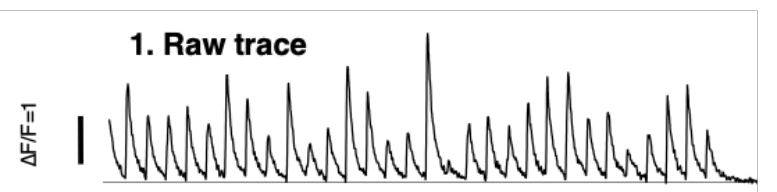

2. Deconvolution

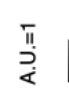
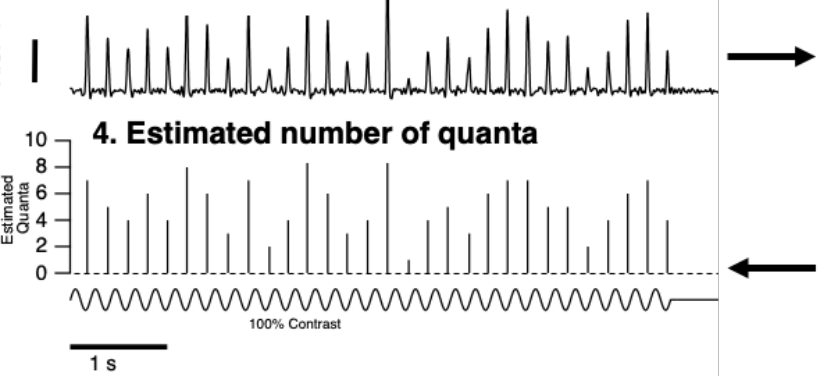

3. Distribution of event amplitudes

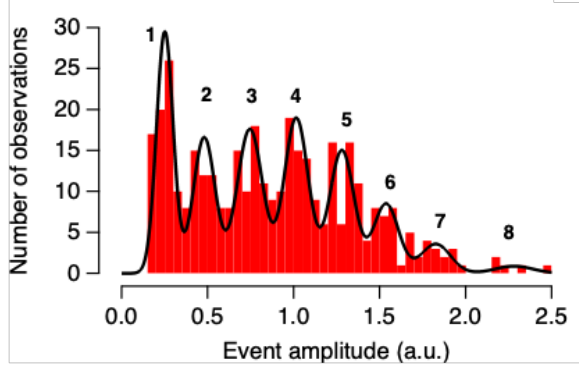

593

594

595

596

597

598

599

600

601

602

603

Figure S3. Decomposition of iGluSnFR signals into vesicle counts

Summary of the basic steps for quantal decomposition of iGluSnFr signals. 1. Raw trace extracted from individual active zones (linescan, $1 \mathrm{KHz}$ ). 2. Deconvolved trace using the estimated Wiener filter. 3. Histogram of event amplitudes for a representative active zone (373 events accumulated using stimulus contrasts of $20 \%, 60 \%$ and $100 \%$ and a frequency of $5 \mathrm{~Hz}$ ). The black line is a fit of eight Gaussians, identified using a Gaussian mixture model. Note that the variance of successive Gaussians did not increase in proportion to the peak number. The first peak had a value of 0.24 , and the distance between peaks averaged 0.25 , indicating the existence of a quantal event equivalent to $\sim 0.25$. .4. Estimation of the number of quanta per event. For more details about analyses see James et al., 2019. 


\section{Methods}

\section{Zebrafish husbandry}

607 Fish were raised and maintained under standard conditions on a $14 \mathrm{~h}$ light/10 $\mathrm{h}$ dark 608 cycle ${ }^{35}$. To aid imaging, fish were heterozygous or homozygous for the casper mutation 609 which results in hypopigmentation and they were additionally treated with1-phenyl-2610 thiourea $(200 \mu \mathrm{M}$ final concentration; Sigma) from 10 hours post fertilization (hpf) to 611 reduce pigmentation. All animal procedures were performed in accordance with the 612 Animal Act 1986 and the UK Home Office guidelines and with the approval of the 613 University of Sussex Animal Welfare and Ethical Review Board. More information about 614 experimental design and reagents is available in the Life Sciences reporting Summary.

617 Experiments were carried out using the following transgenic lines of zebrafish:

618 i) $T g$ (ribeye:;Zf-SyGCaMP2) expressing the synaptically-localized fluorescent calcium 619 reporter SyGCaMP 2.0 in retinal bipolar cells under the ribeye-A promoter ${ }^{24}$.

620 ii) $\operatorname{Tg}\left(-1.8 c t b p 2: G a l 4 V P 16 \_B H\right)$ fish that drive the expression of the transcriptional 621 activator protein Gal4VP16 were generated by co-injection of I-Scel meganuclease and 622 endofree purified plasmid into wild-type zebrafish with a mixed genetic background. A 623 myocardium-specific promoter that drives the expression of mCherry protein was 624 additionally cloned into the plasmid to allow for phenotypical screening of founder fish.

625 iii) $\operatorname{Tg}\left(10 x \cup A S: i G / u S n F R \_M H\right)$ fish driving the expression of the glutamate sensor 626 iGluSnFR under the regulatory control of the $10 \times$ UAS enhancer elements were 627 generated by co-injection of purified plasmid and tol2 transposase RNA into offspring of 628 AB wildtype fish outcrossed to casper wildtype fish. The sequences for the myocardium629 specific promoter driving the expression of enhanced green fluorescent protein (mossy 630 heart) were added to the plasmid to facilitate the screening process. 
iv) $\operatorname{Tg}(-1.8 c t b p 2: S y G C a M P 6)$ fish were generated by co-injection of I-Scel meganuclease and endofree purified plasmid into wild-type zebrafish with a mixed genetic background. The GCaMP6f variant was kindly provided by L. Looger (Janelia Farm). This variant holds a T383S mutation in comparison to the commercially available GCaMP6-fast version (Addgene plasmid 40755).

v) Tg(is/2b:nlsTrpR, tUAS:memGCaMP6f) which drives the expression of memGCaMP6f in the optic tectum was generated by co-injecting pTol2-isl2b-hlsTrpR-pA and $\mathrm{pBH}$-tUAS-memGaMP6f-pA plasmids into single-cell stage eggs. Injected fish were

\section{Multiphoton Imaging In Vivo}

Experiments were carried out in a total of 117 zebrafish larvae (7-9 days postfertilization). Fish were immobilized in $3 \%$ low melting point agarose (Biogene) in E2 medium on a glass coverslip ( 0 thickness) and mounted in a chamber where they were superfused with E2. Imaging was carried out using a two-photon microscope (Scientifica) equipped with a mode-locked titanium-sapphire laser (Chameleon, Coherent) tuned to $915 \mathrm{~nm}$ and an Olympus XLUMPlanFI 20x water immersion objective (NA 0.95). To prevent eye movements, the ocular muscles were paralyzed by injection of $1 \mathrm{~nL}$ of $\alpha$ bungarotoxin $(2 \mathrm{mg} / \mathrm{mL})$ behind the eye. Most imaging was carried out in the dorsal the retina. through both the objective and a sub-stage oil condenser (Olympus, NA 1.4). Emission was filtered through GFP filters (HQ 535/50, Chroma Technology) before detection with GaAsP photomultipliers (H7422P-40, Hamamatsu). The signal from each detector passed through a current-to-voltage converter and then the two signals were added by a summing amplifier before digitization. Scanning and image acquisition were controlled under Scanlmage v.3.6 software ${ }^{52}$. In iGluSnFR recordings images were acquired at $10 \mathrm{~Hz}(128 \times 100$ pixels per frame, $1 \mathrm{~ms}$ per line) while linescans were acquired at $1 \mathrm{kHz}$. 
In GCaMP recordings images were acquired at $20 \mathrm{~Hz}(128 \times 50$ pixels per frame, $1 \mathrm{~ms}$

660 per line). Full-field light stimuli were generated by an amber LED $\left(I_{\max }=590 \mathrm{~nm}\right.$,

661 Thorlabs), filtered through a 590/10 nm BP filter (Thorlabs), and delivered through a light

662 guide placed close to the eye of the fish. These wavelengths will most effectively

663 stimulate red and green cones. The microscope was synchronized to visual stimulation.

664

665 Stimulation protocols

666 Measurements of contrast sensitivity with SyGCaMP2 were made by stimulating the fish

667 with a series of $10 \mathrm{~s}$ stimuli (full-field sinusoidal modulation at $5 \mathrm{~Hz}$ ) around a mean 668 intensity of $55 \mathrm{nW} \mathrm{mm}-2$. Measurements of contrast sensitivity with iGluSnFR used $2 \mathrm{~s}$

669 stimuli. To measure the distribution of events amplitudes and the temporal precision fish

670 were continuously stimulated for $30 \mathrm{~s}$ at a given contrast.

671 Luminance sensitivity was assessed by stimulating the fish with a series of light steps

$672(4 \times 3$ s) at 9 different light intensities increasing in steps of 0.5 log unit steps ranging from

$67311 \mathrm{pW} \mathrm{mm}^{-2}$ to $110 \mathrm{nW} \mathrm{mm}^{-2}$ (equivalent to $3.3 \times 10^{11}$ photons $\mathrm{mm}^{-2}$ ).

675 Drug injections

676 Dopamine signalling was manipulated by injecting the antagonist of D1 receptors SCH

67723390 at a final estimated concentration of $200 \mathrm{nM}$ (Sigma).Finally, the long-lasting 678 dopamine receptor ligand [3H] 2-amino-6,7-dihydroxy 1,2,3,4-tetrahydronapthalene 679 (ADTN) (Sigma) was injected to a final estimated concentration of $200 \mathrm{nM}$. We confirmed 680 that these drugs gained access by including $1 \mathrm{mM}$ Alexa 594 in the injection needle; 681 within 5 mins of injection the dye could be detected within the inner plexiform layer of the 682 retina. Vehicle injection did not affect synaptic responses to varying contrast. 
685 In order to quantify variability in the timing of glutamatergic events, we first calculated the 686 vector strength, $\mathrm{r}_{\mathrm{q}}$, for events composed of q quanta:

$$
r_{q}=\frac{1}{N_{q}} \sqrt{\left(\sum_{i=1}^{N_{q}} \cos \left(\frac{2 \pi t_{q_{i}}}{T}\right)\right)^{2}+\left(\sum_{i=1}^{N_{q}} \sin \left(\frac{2 \pi t_{q_{i}}}{T}\right)\right)^{2}}
$$

where $t_{q i}$ is the time of the $i^{\text {th }} q$-quantal event, $T$ is the stimulus period, and $N_{q}$ is the total number of events of composed of q-quanta. The temporal jitter, $\mathrm{J}_{\mathrm{q}}$, can then be calculated as:

$$
J_{q}=\frac{\sqrt{2\left(1-r_{q}\right)}}{2 \pi f}
$$

where $f$ is the stimulus frequency.

\section{Calculations based on Information Theory}

To quantify the amount of information about a visual stimulus that is contained within the sequence of release events from an active zone we first needed to convert bipolar cell outputs into a probabilistic framework from which we could evaluate the specific information $\left(\mathrm{I}_{2}\right)$, a metric that quantifies how much information about one random variable is conveyed by the observation a specific symbol of another random variable $^{36}$. The time series of quantal events was converted into a probability distribution by dividing into time bins of $20 \mathrm{~ms}$, such that each bin contained either zero events or one event of an integer amplitude. We then counted the number of bins containing events of amplitude 1 , or 2 , or 3 etc. By dividing the number of bins of each type by the total number of bins for each different stimulus, we obtained the conditional distribution of $\mathbf{Q}$ given $\mathbf{S}, p(\boldsymbol{Q} \mid \boldsymbol{S})$, where $\mathbf{Q}$ is the random variable representing the quanta/bin and $\mathbf{S}$ is the random variable representing the stimulus contrasts presented throughout the course of the experiment. In the absence of information about the distribution of contrasts normally experienced by a larval zebrafish, a uniform 

were presented in two different pseudo-random orders, of which one is shown in Fig. 4D. The contrast sensitivity varied between synapses and between morning and afternoon (Fig. 1E-G) so to make allowance for this the stimulus set $\mathrm{S}$ was adjusted for each synapse to span contrasts $\pm 10 \%$ around $C_{1 / 2}$ measured within that synapse. the experimentally defined uniform distribution of stimuli S):

$$
p(S, Q)=p(Q \mid S) p(S)
$$

717 In order to convert this distribution into the conditional distribution of $S$ given $\mathrm{Q}$, we used 718 the definition of the conditional distribution:

$$
p(S \mid Q)=\frac{p(S, Q)}{p(Q)}
$$

From these distributions we computed two metrics: the mutual information $\mathrm{I}(\mathbf{S} ; \mathbf{Q})^{53}$ and specific information $\mathrm{I}_{2}(\mathbf{S} ; \mathbf{q})^{37}$. Mutual information is defined traditionally as:

$$
\begin{gathered}
\mathrm{I}(S ; Q)=H(S)-H(S \mid Q) \\
\mathrm{I}(S ; Q)=\sum_{s \in S} \sum_{q \in Q} p(s, q) \log _{2} \frac{p(s) p(q)}{p(s, q)}=I(Q ; S)
\end{gathered}
$$
stimulus S minus the conditional entropy of the stimulus given the observed symbol in the response q:

$$
I_{2}(S, q)=H(S)-H(S \mid q)
$$

$$
I_{2}(S, q)=-\sum_{s \in S} p(s) \log p(s)+\sum_{s \in S} p(s \mid q) \log p(s \mid q)
$$
representing the amount of information observing each quantal event type $q \in \mathbf{Q}$ carries about the stimulus distribution $\mathbf{S}$. Note that mutual information can also be computed from the specific information as the dot product of the specific information vector $\boldsymbol{I}_{2}$ and the vector describing the probability of an event of a given quantal size $\boldsymbol{p}(\boldsymbol{q})$. This adds to the interpretability of both metrics - the specific information is the amount of information a 
single (specific) symbol gives about the stimulus, and the mutual information is the average amount of information about the stimulus gained from observing any symbol.

Measuring entropy and mutual information from neural responses can be a

737 challenging problem. Estimates require sampling from an unknown discrete probability

738 distribution, and in many cases recording sufficient samples to observe all non-zero 739 probability events is neither tractable nor practical. The biases introduced by

740 undersampling can be a particular problem when the full support of the distribution (all 741 values that map to non-zero probabilities) is high. Within the past few decades, various

742 approaches to correcting biases in information theoretic analyses have been developed ${ }^{54}$.

743 However, as the distributions of interest in this work have both a small support and are

744 well sampled, we have opted to use standard estimates for the quantities of interest.

\section{$746 \quad$ Statistics}

747 All data are given as mean \pm s.e.m. unless otherwise stated in the figure legends. All 748 statistical tests met appropriate assumptions and were calculated using inbuilt 749 functions in IgorPro (Wavemetrics). When data were not normally distributed we used 750 non-parametric tests. Significance was defined as $p<0.05$. Data collection was not 751 randomized because all experiments were carried out within one set of animals.

752 Delivery of different stimuli was randomized where appropriate. Data were only excluded from the analysis if the signal-to-noise ratio (SNR) of the iGluSnFR signals elicited at a given synapse was not sufficient to detect unitary responses to visual stimuli with a SNR of at least three. 


\section{References}

1 Bargmann, C. I. \& Marder, E. From the connectome to brain function. Nat Methods 10, 483490, doi:nmeth.2451 [pii] 10.1038/nmeth.2451 (2013).

2 Kastner, D. B. \& Baccus, S. A. Coordinated dynamic encoding in the retina using opposing forms of plasticity. Nature neuroscience 14, 1317 (2011).

3 Johnston, J. et al. A Retinal Circuit Generating a Dynamic Predictive Code for Oriented Features. Neuron 102, 1211-1222 e1213, doi:10.1016/j.neuron.2019.04.002 (2019).

768

769

770

4 Esposti, F., Johnston, J., Rosa, J. M., Leung, K. M. \& Lagnado, L. Olfactory stimulation selectively modulates the OFF pathway in the retina of zebrafish. Neuron 79, 97-110, doi:10.1016/j.neuron.2013.05.001 (2013).

5 Schroder, S. et al. Arousal Modulates Retinal Output. Neuron 107, 487-495 e489, doi:10.1016/j.neuron.2020.04.026 (2020).

6 Ribelayga, C., Cao, Y. \& Mangel, S. C. The circadian clock in the retina controls rod-cone coupling. Neuron 59, 790-801, doi:10.1016/j.neuron.2008.07.017 (2008).

$7 \mathrm{Li}, \mathrm{L}$. Circadian Vision in Zebrafish: From Molecule to Cell and from Neural Network to Behavior. J Biol Rhythms 34, 451-462, doi:10.1177/0748730419863917 (2019).

8 Zang, J. et al. Circadian regulation of vertebrate cone photoreceptor function. eLife 10, e68903, doi:10.7554/eLife.68903 (2021).

9 Jackson, C. R. et al. Retinal dopamine mediates multiple dimensions of light-adapted vision. The Journal of neuroscience : the official journal of the Society for Neuroscience 32, 9359-9368, doi:10.1523/JNEUROSCI.0711-12.2012 (2012).

10 Euler, T., Haverkamp, S., Schubert, T. \& Baden, T. Retinal bipolar cells: elementary building blocks of vision. Nat Rev Neurosci 15, 507-519, doi:10.1038/nrn3783 (2014).

11 Nikolaev, A., Leung, K. M., Odermatt, B. \& Lagnado, L. Synaptic mechanisms of adaptation and sensitization in the retina. Nat Neurosci 16, 934-941, doi:10.1038/nn.3408 (2013).

12 Zimmermann, M. J. Y. et al. Zebrafish Differentially Process Color across Visual Space to Match Natural Scenes. Curr Biol 28, 2018-2032.e2015, doi:10.1016/j.cub.2018.04.075 (2018).

13 Matsumoto, A. et al. Direction selectivity in retinal bipolar cell axon terminals. Neuron (2021).

14 Lagnado, L. \& Schmitz, F. Ribbon Synapses and Visual Processing in the Retina. Annu Rev Vis Sci 1, 235-262, doi:10.1146/annurev-vision-082114-035709 (2015).

15 Glowatzki, E. \& Fuchs, P. A. Transmitter release at the hair cell ribbon synapse. Nat Neurosci 5, 147-154, doi:10.1038/nn796 (2002).

16 Singer, J. H., Lassova, L., Vardi, N. \& Diamond, J. S. Coordinated multivesicular release at a mammalian ribbon synapse. Nat Neurosci 7, 826-833, doi:10.1038/nn1280 (2004).

17 James, B., Darnet, L., Moya-Diaz, J., Seibel, S. H. \& Lagnado, L. An amplitude code transmits information at a visual synapse. Nat Neurosci 22, 1140-1147, doi:10.1038/s41593-0190403-6 (2019).

18 Lisman, J. E., Raghavachari, S. \& Tsien, R. W. The sequence of events that underlie quantal transmission at central glutamatergic synapses. Nat Rev Neurosci 8, 597-609, doi:10.1038/nrn2191 (2007).

19 Huang, C.-H., Bao, J. \& Sakaba, T. Multivesicular Release Differentiates the Reliability of Synaptic Transmission between the Visual Cortex and the Somatosensory Cortex. The Journal of Neuroscience 30, 11994, doi:10.1523/JNEUROSCI.2381-10.2010 (2010). 
20 Holler, S., Köstinger, G., Martin, K. A. C., Schuhknecht, G. F. P. \& Stratford, K. J. Structure and function of a neocortical synapse. Nature 591, 111-116, doi:10.1038/s41586-020-03134-2 (2021).

21 Shannon, C. A Mathematical Theory of Communication. Bell System Technical Journal 27, 379-423 (1948).

22 Borst, A. \& Theunissen, F. E. Information theory and neural coding. Nat Neurosci 2, 947-957, doi:10.1038/14731 (1999).

23 Marvin, J. S. et al. An optimized fluorescent probe for visualizing glutamate neurotransmission. Nat Methods 10, 162-170, doi:10.1038/nmeth.2333 (2013).

24 Dreosti, E., Odermatt, B., Dorostkar, M. M. \& Lagnado, L. A genetically encoded reporter of synaptic activity in vivo. Nat Methods 6, 883-889, doi:10.1038/nmeth.1399 (2009).

25 Emran, F., Rihel, J., Adolph, A. R. \& Dowling, J. E. Zebrafish larvae lose vision at night. Proceedings of the National Academy of Sciences 107, 6034, doi:10.1073/pnas.0914718107 (2010).

$26 \mathrm{Nie}, \mathrm{K}$. et al. Effects of circadian clock protein Per1b on zebrafish visual functions. Chronobiology international 35, 160-168 (2018).

27 Ashmore, J. \& Copenhagen, D. An analysis of transmission from cones to hyperpolarizing bipolar cells in the retina of the turtle. The Journal of Physiology 340, 569-597 (1983).

28 Faisal, A. A., Selen, L. P. \& Wolpert, D. M. Noise in the nervous system. Nat Rev Neurosci 9 , 292-303, doi:10.1038/nrn2258 (2008).

29 Rusakov, D. A., Savtchenko, L. P. \& Latham, P. E. Noisy Synaptic Conductance: Bug or a Feature? Trends Neurosci 43, 363-372, doi:10.1016/j.tins.2020.03.009 (2020).

30 Berry, M. J., Warland, D. K. \& Meister, M. The structure and precision of retinal spike trains. Proceedings of the National Academy of Sciences 94, 5411, doi:10.1073/pnas.94.10.5411 (1997).

31 Churchland, M. M. et al. Stimulus onset quenches neural variability: a widespread cortical phenomenon. Nature Neuroscience 13, 369-378, doi:10.1038/nn.2501 (2010).

32 Rathbun, D. L., Warland, D. K. \& Usrey, W. M. Spike timing and information transmission at retinogeniculate synapses. Journal of Neuroscience 30, 13558-13566 (2010).

33 Cui, Y., Wang, Y. V., Park, S. J. H., Demb, J. B. \& Butts, D. A. Divisive suppression explains high-precision firing and contrast adaptation in retinal ganglion cells. eLife 5, e19460, doi:10.7554/eLife.19460 (2016).

34 Freed, M. A. Quantal encoding of information in a retinal ganglion cell. J Neurophysiol 94, 1048-1056, doi:10.1152/jn.01276.2004 (2005).

35 Odermatt, B., Nikolaev, A. \& Lagnado, L. Encoding of luminance and contrast by linear and nonlinear synapses in the retina. Neuron 73, 758-773, doi:10.1016/j.neuron.2011.12.023 (2012).

36 Stone, J. V. Principles of Neural Information Theory: Computational Neuroscience and Metabolic Efficiency (Sebtel Press, 2018).

37 DeWeese, M. R. \& Meister, M. How to measure the information gained from one symbol. Network 10, 325-340 (1999).

38 Attwell, D. \& Laughlin, S. B. An energy budget for signaling in the grey matter of the brain. Journal of Cerebral Blood Flow \& Metabolism 21, 1133-1145 (2001).

39 Harris, Julia J., Jolivet, R. \& Attwell, D. Synaptic Energy Use and Supply. Neuron 75, 762777, doi:https://doi.org/10.1016/j.neuron.2012.08.019 (2012).

40 Koch, K. et al. How much the eye tells the brain. Curr Biol 16, 1428-1434, doi:10.1016/j.cub.2006.05.056 (2006). 

receptor immunolocalization in goldfish retina. Journal of Comparative Neurology 411, 705-714 (1999). receptor expression is bipolar cell type-specific in the mouse retina. The Journal of comparative neurology 524, 2059-2079, doi:10.1002/cne.23932 (2016).

43 Ribelayga, C., Wang, Y. \& Mangel, S. C. A circadian clock in the fish retina regulates dopamine release via activation of melatonin receptors. The Journal of Physiology 554, 467-482, doi:https://doi.org/10.1113/jphysiol.2003.053710 (2004).

44 Moya-Díaz, J., James, B. \& Lagnado, L. Modulation of the vesicle code transmitting the visual signal in the retina. bioRxiv, 2020.2004.2022.056119, doi:10.1101/2020.04.22.056119 (2020).

861

862

45 Thermos, K. Functional mapping of somatostatin receptors in the retina: a review. Vision Research 43, 1805-1815, doi:https://doi.org/10.1016/S0042-6989(03)00169-X (2003).

864

865

46 Freed, M. A. \& Liang, Z. Synaptic noise is an information bottleneck in the inner retina during dynamic visual stimulation. The Journal of physiology 592, 635-651, doi:10.1113/jphysiol.2013.265744 (2014).

866

47 McDonnell, M. D. \& Ward, L. M. The benefits of noise in neural systems: bridging theory and

867 experiment. Nature Reviews Neuroscience 12, 415-425, doi:10.1038/nrn3061 (2011).

869

48 Christie, J. M. \& Jahr, C. E. Multivesicular release at Schaffer collateral-CA1 hippocampal synapses. The Journal of neuroscience : the official journal of the Society for Neuroscience 26, 210-216, doi:10.1523/JNEUROSCI.4307-05.2006 (2006).

871

49 Auger, C., Kondo, S. \& Marty, A. Multivesicular release at single functional synaptic sites in cerebellar stellate and basket cells. The Journal of neuroscience : the official journal of the Society for Neuroscience 18, 4532-4547 (1998).

50 Higley, M. J., Soler-Llavina, G. J. \& Sabatini, B. L. Cholinergic modulation of multivesicular release regulates striatal synaptic potency and integration. Nature neuroscience 12, 1121-1128 (2009).

51 Chalifoux, J. R. \& Carter, A. G. GABAB receptors modulate NMDA receptor calcium signals in dendritic spines. Neuron 66, 101-113 (2010). laser scanning microscopes. Biomed Eng Online 2, 13, doi:10.1186/1475-925X-2-13 (2003).

883

53 de Ruyter van Steveninck, R. R., Lewen, G. D., Strong, S. P., Koberle, R. \& Bialek, W. Reproducibility and variability in neural spike trains. Science 275, 1805-1808, doi:10.1126/science.275.5307.1805 (1997).

88454 Pola, G., Schultz, S. R, Petersen, R. S \& Panzeri, S. in Neuroscience Databases: A Practical

885 Guide (Springer, Boston, MA, 2003).

886 